\title{
Dynamics of long gas bubbles rising in a vertical tube in a co-current liquid flow
}

\author{
M. Magnini, ${ }^{1,2, *}$ S. Khodaparast,${ }^{1,3}$ O. K. Matar, ${ }^{1}$ H. A. Stone,${ }^{3}$ and J. R. Thome ${ }^{2}$ \\ ${ }^{1}$ Department of Chemical Engineering, \\ Imperial College London, London SW7 2AZ, United Kingdom \\ ${ }^{2}$ Laboratory of Heat and Mass Transfer, \\ Ecole Polytechnique Fédérale de Lausanne, EPFL-STI-IGM-LTCM, \\ Station 9, CH-1015 Lausanne, Switzerland \\ ${ }^{3}$ Department of Mechanical and Aerospace Engineering, \\ Princeton University, Princeton, New Jersey 08544, USA
}




\begin{abstract}
When a confined long gas bubble rises in a vertical tube in a co-current liquid flow, its translational velocity is the result of both buoyancy and mean motion of the liquid. A thin film of liquid is formed on the tube wall and its thickness is determined by the interplay of viscous, inertial, capillary and buoyancy effects, as defined by the values of the Bond number (Bo $\equiv \rho g R^{2} / \sigma$ with $\rho$ being the liquid density, $g$ the gravitational acceleration, $R$ the tube radius and $\sigma$ the surface tension $)$, capillary number $\left(\mathrm{Ca}_{\mathrm{b}} \equiv \mu U_{b} / \sigma\right.$ with $U_{b}$ being the bubble velocity and $\mu$ the liquid dynamic viscosity) and Reynolds number $\left(\operatorname{Re}_{\mathrm{b}} \equiv 2 \rho U_{b} R / \mu\right)$. We perform experiments and numerical simulations to investigate systematically the effect of buoyancy $(\mathrm{Bo}=0-5)$ on the shape and velocity of the bubble and on the thickness of the liquid film for $\mathrm{Ca}_{\mathrm{b}}=10^{-3}-10^{-1}$ and $\mathrm{Re}_{\mathrm{b}}=10^{-2}-10^{3}$. A theoretical model, based on an extension of Bretherton's lubrication theory, is developed and utilized for parametric analyses; its predictions compare well with the experimental and numerical data. This study shows that buoyancy effects on bubbles rising in a co-current liquid flow make the liquid film thicker and the bubble rise faster, when compared to the negligible gravity case. In particular, gravitational forces impact considerably the bubble dynamics already when Bo $<0.842$, with $\mathrm{Bo}_{\mathrm{cr}}=0.842$ being the critical value below which a bubble does not rise in a stagnant liquid in a circular tube. The liquid film thickness and bubble velocity in a liquid co-flow may vary by orders of magnitude as a result of small changes of Bo around this critical value. The reduction of the liquid film thickness for increasing values of the Reynolds numbers, usually observed for $\mathrm{Re}_{\mathrm{b}} \lesssim 10^{2}$ when Bo $\ll 1$, becomes more evident at larger Bond numbers. Buoyancy effects also have a significant influence on the features of the undulation appearing near the rear meniscus of the bubble, as they induce a substantial increase in its amplitude and decrease in its wavelength.
\end{abstract}

\footnotetext{
*m.magnini@imperial.ac.uk
} 


\section{INTRODUCTION}

The flow of an elongated gas bubble in a narrow gap is of interest to many diverse processes that span different science and engineering fields, e.g. microchannel two-phase cooling [1], transport of pollutants in unsaturated soil [2], cleaning of bacterial cells from medical surfaces [3], medical therapy [4] and membrane-less electrolyzers for hydrogen production [5]. As the bubble progresses through a liquid-filled duct, capillary and viscous forces promote the formation of a thin film of liquid between the liquid-gas interface and the channel wall and its thickness impacts the wall-fluid exchanges. Manifestations of this are exemplified by the fact that the heat transfer coefficient in a slug flow boiling regime is inversely proportional to this thickness [6], while the mass transfer from the gas to the walls of a multiphase monolith reactor is enhanced by a short diffusion path [7, 8]. Gas bubbles generated in water-saturated soil are effective in detaching micron-sized colloids adhering on the pore walls due to surface tension forces, if the liquid film is thinner than the particle size [9]. In medical practice, air bubbles are introduced in pulmonary airways by mechanical ventilation of a collapsed lung, and the consequent shear stress exerted on the airway walls may injure the lung tissues [10]. Therefore, the ability to predict the dynamics of long bubbles traveling in a confined geometry attracts attention from a diverse range of research fields.

The present work focuses on vertically-oriented channels with a circular cross-section. When a long gas bubble travels upward in a vertical tube in the presence of a co-current liquid flow, its translational velocity, $U_{b}$, is a superposition of the bubble velocity in a stagnant liquid $U_{b, 0}$ and a contribution due to the liquid flow rate [11]:

$$
U_{b}=U_{b, 0}+\Gamma U_{l}
$$

where $U_{l}$ is the mean liquid velocity in the tube and $\Gamma$ is an empirical coefficient that depends on the dimensionless parameters of the problem. Within the assumption of an axisymmetric flow, the thickness of the liquid film surrounding the bubble is constant along the perimeter of the tube, as shown in the schematic depicted in Fig. 1. The impact of buoyancy on the dynamics of the rising bubble may be quantified by the Bond number, Bo $\equiv \rho g R^{2} / \sigma$, where $\rho$ denotes the liquid density (the density of the gas is considered negligible in this work), $g$

the gravitational acceleration, $R$ the tube radius and $\sigma$ the surface tension. The dynamics of long gas bubbles translating in a vertical tube has been investigated extensively in the limits 
Table I. Selected studies on the dynamics of isolated long bubbles in co-current liquid flows and circular tubes. References on bubble-trains or bubbles rising in a stagnant liquid are not considered here. The nondimensional groups appearing below are defined as follows: Bo $\equiv \rho g R^{2} / \sigma, \mathrm{Ca}_{\mathrm{b}} \equiv$ $\mu U_{b} / \sigma, \operatorname{Re}_{\mathrm{b}} \equiv 2 \rho U_{b} R / \mu, \operatorname{Re}_{\mathrm{l}} \equiv 2 \rho U_{l} R / \mu$ and $\mathrm{We}_{\mathrm{b}} \equiv \mathrm{Ca}_{\mathrm{b}} \operatorname{Re}_{\mathrm{b}}$

Bo $\ll 1$

\begin{tabular}{|c|c|c|c|}
\hline Reference & Flow parameters & Methodology & Main observations \\
\hline Bretherton [12] & $\begin{aligned} \mathrm{Ca}_{\mathrm{b}} & \leq 0.005 \\
\operatorname{Re}_{\mathrm{b}} & \ll 1\end{aligned}$ & $\begin{array}{l}\text { Lubrication theory applied to } \\
\text { the flow in the liquid film }\end{array}$ & $\frac{h_{0}}{R}=1.34 \mathrm{Ca}_{\mathrm{b}}^{2 / 3}$ \\
\hline Taylor [13] & $\begin{array}{c}\mathrm{Ca}_{\mathrm{b}} \text { up to } 2 \\
\mathrm{Re}_{\mathrm{b}} \ll 1\end{array}$ & $\begin{array}{l}\text { Experimental measurement } \\
\text { of liquid film thickness }\end{array}$ & $h_{0} \rightarrow R / 3$ as $\mathrm{Ca}_{\mathrm{b}} \rightarrow 2$ \\
\hline Aussillous and Quéré [14] & $\begin{aligned} \mathrm{Ca}_{\mathrm{b}} & \lesssim 2 \\
\mathrm{Re}_{\mathrm{b}} & \ll 1\end{aligned}$ & $\begin{array}{l}\text { Experimental measurements } \\
\text { and fit to Taylor's data }\end{array}$ & $\frac{h_{0}}{R}=\frac{1.34 \mathrm{Ca}_{\mathrm{b}}{ }^{2 / 3}}{1+3.35 \mathrm{Ca}_{\mathrm{b}}^{2 / 3}}$ \\
\hline de Ryck [15] & $\begin{array}{c}\mathrm{Ca}_{\mathrm{b}} \leq 2 \\
\operatorname{Re}_{\mathrm{b}} \lesssim 10^{3}\end{array}$ & $\begin{array}{l}\text { Lubrication theory } \\
\text { including inertia }\end{array}$ & $\begin{array}{l}\text { Data of }[13] \text { and }[14] \\
\text { well predicted by the model }\end{array}$ \\
\hline Han and Shikazono [16] & $\begin{aligned} \mathrm{Ca}_{\mathrm{b}} & \leq 0.2 \\
\operatorname{Re}_{\mathrm{b}} & \lesssim 10^{3}\end{aligned}$ & $\begin{array}{c}\text { Experimental measurement } \\
\text { of liquid film thickness }\end{array}$ & $\frac{h_{0}}{R} \approx \frac{\mathrm{Ca}_{\mathrm{b}}{ }^{2 / 3}}{1+\mathrm{Ca}_{\mathrm{b}}{ }^{2 / 3}+f\left(\mathrm{Ca}_{\mathrm{b}}, \mathrm{Re}_{\mathrm{b}}\right)-g\left(\mathrm{We}_{\mathrm{b}}\right)}$ \\
\hline Magnini et al. $[17]$ & $\begin{aligned} \mathrm{Ca}_{\mathrm{b}} & \leq 0.1 \\
\operatorname{Re}_{\mathrm{b}} & \leq 10^{3}\end{aligned}$ & $\begin{array}{l}\text { Lubrication theory and } \\
\text { numerical simulations }\end{array}$ & $\begin{array}{l}\text { When } \mathrm{We}_{\mathrm{b}} \geq 1 \text {, undulations appear } \\
\text { at the rear meniscus and } \lambda=\lambda\left(\mathrm{We}_{\mathrm{b}}\right)\end{array}$ \\
\hline
\end{tabular}

Bo $\gg 1$, vertical tubes

\begin{tabular}{|c|c|c|c|}
\hline Reference & Flow conditions & Study performed & Main observations \\
\hline Nicklin et al. [11] & $\begin{array}{c}\mathrm{Re}_{1} \text { up to } 60000 \\
\text { Bo }=22\end{array}$ & $\begin{array}{l}\text { Experimental measurement } \\
\text { of bubble velocity }\end{array}$ & $\begin{array}{c}U_{b, 0}=0.35(2 g R)^{1 / 2} . \Gamma=1.2 \\
\text { for } \operatorname{Re}_{1}>8000, \Gamma \rightarrow 1.8 \text { as } U_{l} \rightarrow 0\end{array}$ \\
\hline Collins et al. [18] & $\begin{array}{l}\operatorname{Re}_{1} \text { up to } 20000 \\
\text { Bo }>90\end{array}$ & $\begin{array}{l}\text { Potential flow theory } \\
\text { and experiments }\end{array}$ & $\begin{array}{c}\Gamma=2.27 \text { if laminar flow } \\
\text { and } \Gamma=1.2-1.4 \text { if turbulent flow }\end{array}$ \\
\hline Bendiksen [19] & $\begin{aligned} \operatorname{Re}_{\mathrm{b}} & >100 \\
\text { Bo } & >10\end{aligned}$ & $\begin{array}{c}\text { Potential flow theory including } \\
\text { surface tension effects }\end{array}$ & $\begin{array}{c}\text { Both } U_{b, 0} \text { and } \Gamma \text { decrease as } \\
\text { Bo decreases }\end{array}$ \\
\hline
\end{tabular}

Bo $\sim 1$, vertical tubes

\begin{tabular}{|c|c|c|c|}
\hline Reference & Flow conditions & Study performed & Main observations \\
\hline Bretherton [12] & $\begin{array}{c}\mathrm{Ca}_{\mathrm{b}} \leq 0.005, \\
\mathrm{Re}_{\mathrm{b}} \ll 1\end{array}$ & $\begin{array}{c}\text { Lubrication theory applied to } \\
\text { the flow in the liquid film }\end{array}$ & $\begin{array}{c}h_{0} / R \text { is increased by a factor } \\
1 \pm \frac{2}{3} \text { Bo for upward/downward flow }\end{array}$ \\
\hline Thulasidas et al. $[7]$ & $\begin{array}{c}0.004 \leq \mathrm{Ca}_{\mathrm{b}} \leq 3, \\
\mathrm{Re}_{\mathrm{b}} \leq 2, \mathrm{Bo}= \pm 0.43\end{array}$ & $\begin{array}{c}\text { Experimental measurement } \\
\text { of liquid film thickness }\end{array}$ & $\begin{array}{c}h_{0} \text { at the lowest } \mathrm{Ca}_{\mathrm{b}} \text { tested does not agree } \\
\text { with Bretherton's correction factor }\end{array}$ \\
\hline
\end{tabular}

Bo $\gg 1$ (large tubes), and Bo $\ll 1$, where gravity effects vanish and the pipe orientation is not important. A summary of the most relevant works is provided in Table I.

The Bo $\ll 1$ regime (where $U_{b, 0}=0$ ) is pertinent to flow in tubes with radii smaller than the capillary length $\sqrt{\sigma /(\rho g)}$, e.g. the flow of a bubble in an aqueous solution with $\rho=1000 \mathrm{~kg} / \mathrm{m}^{3}, \sigma=0.073 \mathrm{~N} / \mathrm{m}$ in a tube of diameter $2 R=1 \mathrm{~mm}$ gives $\mathrm{Bo}=0.03$ at 
terrestrial gravity, and it was originally studied as a model for enhanced oil recovery and flow in porous media [20]. In this regime, the bubble dynamics is fully determined by the interplay between viscous and capillary forces, characterized by the bubble capillary number $\mathrm{Ca}_{\mathrm{b}} \equiv \mu U_{b} / \sigma$ (with $\mu$ being the liquid dynamic viscosity), and by the ratio of inertial to viscous effects expressed by the bubble Reynolds number, $\operatorname{Re}_{\mathrm{b}} \equiv 2 \rho U_{b} R / \mu$. In the viscocapillary regime $\mathrm{Re}_{\mathrm{b}} \ll 1$, the theoretical analysis of Bretherton [12] was based on the model of an annular liquid film region separating the front and rear menisci of the bubble, where in the limit that $\mathrm{Ca}_{\mathrm{b}} \lesssim 10^{-3}$ the thickness of the film can be predicted as $h_{0} / R=1.34 \mathrm{Ca}_{\mathrm{b}}{ }^{2 / 3}$. Later, Aussillous and Quéré [14] performed a scaling analysis of the forces acting on the front meniscus of the bubble and utilized the film thickness measurements of Taylor [13] for very viscous liquids to set an empirical constant in their scaling law. In the visco-inertial regime $\mathrm{Re}_{\mathrm{b}} \gg 1$, inertial forces tend to reduce the liquid film thickness at intermediate values of the Reynolds number $\left(\operatorname{Re}_{\mathrm{b}} \lesssim 10^{2}\right)$ and to increase it when $\operatorname{Re}_{\mathrm{b}}>10^{2}[8,16]$. The theoretical models of de Ryck [15] and Magnini et al. [17] adopted a lubrication approximation to describe the dynamics of the free-surface in the presence of inertia and derived a third-order ordinary differential equation for the liquid film thickness; solution of this equation yielded the entire profiles of the front and rear menisci of the bubble.

The $\mathrm{Bo} \gg 1$ regime is obtained in large vertical tubes $(\mathrm{Bo}>10$ for an aqueous solution in a tube of diameter $2 R>20 \mathrm{~mm}$ ) and it was originally motivated by submarine applications $[21,22]$. The main focus of the studies in this regime was on the determination of $U_{b, 0}$ and of the coefficient $\Gamma$ in Eq. (1) for co-current flows. The first study for co-current flow conditions was apparently performed by Nicklin et al. [11], who reported values of $\Gamma=1.2$ for turbulent flows $\left(\operatorname{Re}_{1} \equiv 2 \rho U_{l} R / \mu>8000\right)$ and values converging to about 1.8 as the liquid flow rate was reduced to zero. Theoretical analyses were limited to the inertia-controlled regime, which was assumed to occur when $\operatorname{Re}_{\mathrm{b}}>105$ and $\mathrm{Bo}>25$ [18]. Collins et al. [18] adopted potential flow theory to study the inviscid axisymmetric flow of liquid around the bubble nose, which was assumed spherical. They obtained the theoretical values of $\Gamma=2.27$ for laminar flow $\left(\operatorname{Re}_{\mathrm{l}}<2100\right)$ and $\Gamma=1.2-1.4$ for turbulent flow $\left(\operatorname{Re}_{\mathrm{l}}>2100\right)$ which matched their experimental data well. Bendiksen [19] included the effect of surface tension on the bubble profile in the theory developed by Collins et al. [18], and proposed a correlation to evaluate $\Gamma$ as a function of Bo for Bo $>10$. More recently, new empirical correlations have been obtained to estimate $\Gamma$, albeit in the limit of large tubes and large Reynolds numbers 
$[23-25]$

In contrast, the vertical flow of individual long bubbles in co-current liquid for Bo $\sim 1$ has received far less attention. This is now of interest for a number of applications such as compact heat exchangers, where the refrigerants utilized (hydro-fluoro-carbons and hydrofluoro-olefins) have values of the liquid density marginally above that of water but much smaller surface tensions, typically in the range of $\sigma=0.01-0.02 \mathrm{~N} / \mathrm{m}$, thus giving Bond numbers within the range $\mathrm{Bo}=0.1-5$ for tubes of diameter $2 R=1-5 \mathrm{~mm}$. In the low capillary number limit, Bretherton [12] proposed to correct the film thickness by a factor $1 \pm \frac{2}{3}$ Bo for upward and downward flow, respectively. He compared this corrected scaling with the results of experiments performed in a vertical tube, however the scatter of the data available makes it difficult to draw clear conclusions regarding the validity of the correction term. Thulasidas et al. [7] performed experiments in vertical capillaries with isolated bubbles at $\mathrm{Bo}=0.43$ by systematically varying $\mathrm{Ca}_{\mathrm{b}}$. They observed that, at the lowest experimental $\mathrm{Ca}_{\mathrm{b}}\left(\mathrm{Ca}_{\mathrm{b}} \approx 0.01\right)$, the film thicknesses for both upward and downward flow did not agree with Bretherton's correction term when compared to the horizontal flow case. The same observation was reported in the numerical study of Hazel and Heil [26] with Bo $=0.45$. Also, it is worthwhile to mention that, in the second part of his classical paper, Bretherton [12] modified his theory to analyze the upward motion of a long bubble in a stagnant liquid and observed that no steady solution for the profile of the bubble nose exists if the Bond number is below a critical value, $\mathrm{Bo}_{\mathrm{cr}}=0.842$, as confirmed in other experimental studies [27-29]. This critical value of the Bond number is often assumed as the lower limit for the importance of gravitational effects also in the presence of a bulk liquid flow [30].

The objective of the present work is to investigate the dynamics of individual long bubbles rising in a vertical circular channel in a co-current laminar liquid flow, for values of the Bond number around the critical condition $\mathrm{Bo}_{\mathrm{cr}}=0.842$. In particular, we assess the influence of buoyancy effects when the Bond number transitions across the critical value $(\mathrm{Bo}=0-5)$. This study adopts a combination of theory, experiments and numerical simulations. The theoretical model is based on an extension of the Bretherton theory to describe the flow in the liquid film surrounding the bubble, and incorporates the effects of capillary, viscous, inertial and gravitational forces. The experiments are based on a refractive-index-matching technique, and are employed for a quantitative analysis of the bubble shape and velocity in the $\operatorname{Re}_{\mathrm{b}} \ll 1$ regime. The numerical simulations are based on a Volume-Of-Fluid (VOF) 
method [31], and are utilized to explore the $\mathrm{Re}_{\mathrm{b}} \gg 1$ regime. Experiments and numerical simulations also provide validation to the theoretical model. The rest of this article is organized as follows: in Sec. II, a description of the flow problem is presented; the results of the theoretical and experimental analyses for flows with negligible inertia are discussed in Sec. III; in Sec. IV, the outcome of the theoretical model and numerical simulations for flows with inertial effects are described.

\section{PROBLEM FORMULATION}

We consider an elongated gas bubble rising at a steady velocity $U_{b}$ in a vertical channel of circular cross-section of radius $R$. The tube is filled with liquid, which flows with an average velocity denoted $U_{l}$ in the same direction as the bubble. The flow is subject to a gravitational force acting downward with acceleration $g$, so that buoyancy contributes to the upward motion of the bubble. Figure 1 shows a sketch of the flow configuration under consideration, in a reference frame attached to the gas bubble. The bubble profile and the flow field are assumed to be axisymmetric. In order to describe the thin liquid film, the radial direction $y$ is chosen from the tube wall inwards. The axial coordinate $x$ is directed upward, and the reference $x=0$ will be changed, as convenient, during this work. In these coordinates, the axial and radial liquid velocity components are defined as $u$ and $v$, respectively, with $v$ being positive when directed towards the axis of the tube. The walls move with velocity $(u, v)=\left(-U_{b}, 0\right)$, in accordance with the no-slip and no-penetration conditions. At steady-state, the gas-liquid interface is located at $h(x)$.

Following the notation and description of Bretherton [12], we assume that there exists a region of uniform film thickness, region $C D$ in Fig. 1, where the liquid moves parallel to the wall of the tube and the film has a uniform film thickness $h_{0}$. From point $C$, moving towards its nose, the bubble presents a front dynamic meniscus region indicated as $A C$, with $A$ identified as the intersection between the interface profile and the axis of the pipe. Here, the liquid film thickness increases monotonically from $h=h_{0}$ at $C$ to $h=R$ at $A$. From point $D$, moving towards the bubble rear, there exists a dynamic meniscus region $D F$ where the liquid film thickness first undulates around $h_{0}[17]$ then eventually grows monotonically to $h=R$ at point $F$, thus forming the rear cap.

We assume that the flow is laminar, steady, and incompressible, and that the fluid is 


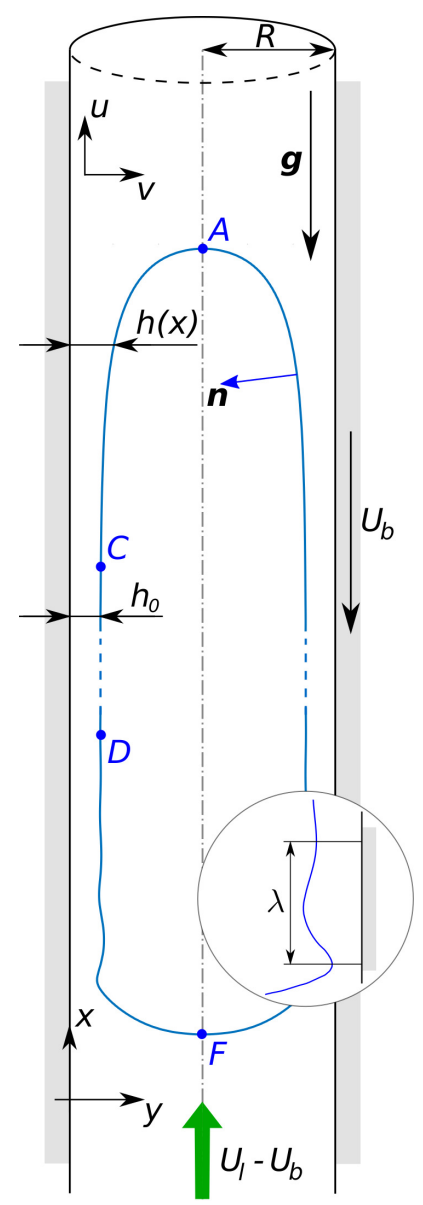

Figure 1. Sketch of a confined elongated bubble flowing within a vertical tube and notation used in this work. Regions $A C$ and $D F$ represent the front and rear menisci, respectively; region $C D$ represents the uniform film zone. Points $B$ and $E$, which in the original work of Bretherton [12] defined static menisci regions $A B$ (front) and $E F$ (rear), are not included in the figure because under the conditions presently studied the bubble profile does not necessarily end with two static menisci regions.

Newtonian. The steady-state Navier-Stokes equations governing the flow in the liquid film surrounding the bubble are reported in Appendix A in dimensional form. We nondimensionalize according to:

$$
\widehat{u}=\frac{u}{U}, \quad \widehat{v}=\frac{v}{V}, \quad \widehat{x}=\frac{x}{\ell}, \quad \widehat{y}=\frac{y}{h_{0}}, \quad \widehat{p}=\frac{p}{\mu U \ell / h_{0}^{2}}, \quad \widehat{h}=\frac{h}{h_{0}}, \quad \widehat{\kappa}=\frac{\kappa}{h_{0} / \ell^{2}},
$$

where $p$ denotes the pressure, $\mu$ the dynamic viscosity of the liquid and $\kappa$ the interface curvature. We assume that the uniform film thickness $h_{0}$ is much smaller than the characteristic 
length of the dynamic meniscus $\ell$, i.e. $\epsilon=h_{0} / \ell \ll 1$, so that from the continuity equation it follows that $V=\epsilon U$. The bubble velocity $U_{b}$ is used hereafter as a velocity scale, so that $U=U_{b}$. We now suppress the hat decoration. The dimensionless Navier-Stokes equations, written in the axisymmetric, cylindrical coordinates introduced in Fig. 1, are given by

$$
\begin{aligned}
u_{x}+v_{y}-v \frac{H}{1-H y} & =0 \\
\frac{\epsilon \operatorname{Re}_{\mathrm{b}} H}{2}\left(u u_{x}+v u_{y}\right) & =-p_{x}+\epsilon^{2} u_{x x}+u_{y y}-u_{y} \frac{H}{1-H y}-\frac{\mathrm{Bo}^{2}}{\mathrm{Ca}_{\mathrm{b}}} \\
\frac{\epsilon^{3} \operatorname{Re}_{\mathrm{b}} H}{2}\left(u v_{x}+v v_{y}\right) & =-p_{y}+\epsilon^{4} v_{x x}+\epsilon^{2} v_{y y}-\epsilon^{2} v_{y} \frac{H}{1-H y}-\epsilon^{2} v \frac{H^{2}}{(1-H y)^{2}}
\end{aligned}
$$

where the subscripts indicate derivatives and the nondimensional groups are

$$
H \equiv \frac{h_{0}}{R}, \quad \mathrm{Ca}_{\mathrm{b}} \equiv \frac{\mu U_{b}}{\sigma}, \quad \operatorname{Re}_{\mathrm{b}} \equiv \frac{2 \rho U_{b} R}{\mu}, \quad \text { Вo } \equiv \frac{\rho g R^{2}}{\sigma},
$$

with $\rho$ being the liquid density and $\sigma$ the surface tension. The no-slip and no-penetration conditions apply at the channel wall:

$$
u=-1 \quad \text { and } \quad v=0, \text { at } \quad y=0 \text {. }
$$

When neglecting the viscous stress within the gas phase, and setting the pressure within the bubble to a zero reference value, the tangential and normal-stress conditions at the interface are given by

$$
\begin{aligned}
& u_{y}+\epsilon^{2} v_{x}+\frac{2 \epsilon^{2} h_{x}}{1-\epsilon^{2} h_{x}^{2}}\left(v_{y}-u_{x}\right)=0, \quad \text { at } y=h(x), \\
& \text { and } p-2 \epsilon^{2} v_{y}+\left(\epsilon^{2} u_{y}+\epsilon^{4} v_{x}\right) h_{x}=-\frac{\epsilon^{3}}{\mathrm{Ca}_{\mathrm{b}}} \kappa, \quad \text { at } y=h(x) .
\end{aligned}
$$

The unit normal vector at the interface is directed into the bubble (see Fig. 1) and has components

$$
\boldsymbol{n}=\frac{1}{\left(1+\epsilon^{2} h_{x}^{2}\right)^{1 / 2}}\left(-\epsilon h_{x}, 1\right),
$$

and the interface curvature is

$$
\kappa=\kappa_{1}+\kappa_{2}=\frac{h_{x x}}{\left(1+\epsilon^{2} h_{x}^{2}\right)^{3 / 2}}+\frac{H}{\epsilon^{2}} \frac{1}{(1-H h)\left(1+\epsilon^{2} h_{x}^{2}\right)^{1 / 2}},
$$

where $\kappa_{1}$ indicates the curvature in the $x-y$ plane and $\kappa_{2}$ the contribution due to the axial symmetry. 


\section{FLOWS WITH NEGLIGIBLE INERTIA}

In this section, we study the dynamics of the bubble in the $\mathrm{Re}_{\mathrm{b}} \ll 1$ regime. Sections III A and B illustrate the theoretical model; the experimental setup adopted to analyze the bubble dynamics in the absence of inertial effects is described in Sec. III C; the results obtained with the model and experiments are compared and discussed in Sections IIID and E.

\section{A. Approximate model}

We develop a model to derive the shape of the front meniscus of the rising bubble, based on the numerical solution of an approximate set of equations governing the steady flow in the liquid film. Starting from the complete nondimensional equations formulated in Section II, we assume that $\epsilon=h_{0} / \ell \ll 1$. The capillary number is considered to scale as $\mathrm{Ca}_{\mathrm{b}}=\mathcal{O}\left(\epsilon^{3}\right)$, so that it follows from Eq. (7) at leading-order that pressure and surface tension forces are balanced at the interface. This choice determines the scaling of the length of the dynamic meniscus, $\ell=h_{0} \mathrm{Ca}_{\mathrm{b}}{ }^{-1 / 3}$. According to the theory of Bretherton [12] for $\mathrm{Ca}_{\mathrm{b}} \ll 1$, we set the nondimensional film thickness to scale as $H=\mathcal{O}\left(\epsilon^{2}\right)$. In this section, we neglect the contribution of inertial forces by assuming $\mathrm{Re}_{\mathrm{b}}=\mathcal{O}(1)$ or smaller. We assume that Bo $=\mathcal{O}\left(\epsilon^{-1}\right)$, so that the buoyancy term in Eq. (3b) appears with the same order as the streamwise pressure gradient. At leading-order in $\epsilon$, Eq. (3) becomes

$$
\begin{aligned}
u_{x}+v_{y} & =0, \\
u_{y y} & =p_{x}+T^{2}, \\
p_{y} & =0,
\end{aligned}
$$

where

$$
T^{2} \equiv H^{2} \frac{\mathrm{Bo}}{\mathrm{Ca}_{\mathrm{b}}}
$$

At the tube wall, the no-slip and no-penetration boundary conditions expressed in Eq. (5) apply. The boundary conditions at the gas-liquid interface are rewritten from Eqs. (6) and (7) at leading-order in $\epsilon$ :

$$
\begin{aligned}
& u_{y}=0, \quad \text { at } y=h(x), \\
& \text { and } p=-\kappa, \quad \text { at } y=h(x) .
\end{aligned}
$$


A consistent leading-order expression for the interface curvature is $\kappa=h_{x x}+H / \mathrm{Ca}_{\mathrm{b}}{ }^{2 / 3}$, which is accurate in the thin-film region, where $\left|h_{x}\right| \ll 1$. However, near the bubble front and rear caps, $\left|h_{x}\right|$ becomes large. Therefore, to obtain a set of equations that describe the liquid flow both in the thin-film and the bubble caps regions, we retain the complete expression of the interface curvature $[32,33]$

$$
\kappa=\frac{h_{x x}}{\left(1+\mathrm{Ca}_{\mathrm{b}}{ }^{2 / 3} h_{x}^{2}\right)^{3 / 2}}+\frac{H}{\mathrm{Ca}_{\mathrm{b}}{ }^{2 / 3}} \frac{1}{(1-H h)\left(1+\mathrm{Ca}_{\mathrm{b}}{ }^{2 / 3} h_{x}^{2}\right)^{1 / 2}} .
$$

By integrating once the $y$-momentum Eq. (10c) along $y$ and using the boundary condition Eq. (13) to fix the integration constant, the pressure gradient along $x$ in Eq. (10b) can be written as $p_{x}=-\kappa_{x}$. Integrating the $x$-momentum equation twice along $y$, with the boundary conditions expressed in Eqs. (5) and (12), the velocity profile in the dynamic meniscus is derived:

$$
u(x, y)=\left(-\kappa_{x}+T^{2}\right)\left(\frac{y^{2}}{2}-h y\right)-1 .
$$

At steady-state, the volume-flux of liquid across the dynamic meniscus region $A C$ equals the flux of liquid across the flat-film region $C D$ :

$$
Q=\int_{0}^{h} u(x, y) d y=\int_{0}^{1} u_{C D}(y) d y
$$

where $u_{C D}$ is the liquid velocity profile in the flat-film region

$$
u_{C D}(y)=T^{2}\left(\frac{y^{2}}{2}-y\right)-1 .
$$

Introducing Eqs. (15) and (17) into Eq. (16), calculating the integrals and rearranging the terms, a third-order nonlinear ordinary differential equation (ODE) for the liquid film profile is obtained:

$$
\kappa_{x}=\underbrace{3 \frac{(h-1)}{h^{3}}}_{\text {viscous term }}+\underbrace{T^{2} \frac{\left(h^{3}-1\right)}{h^{3}}}_{\text {buoyancy term }} .
$$

In Eq. (18), the first term on the right-hand side is the standard leading-order viscous term that enters the Bretherton problem, while the last term introduces the gravitational force. Using the complete expression of the interface curvature shown in Eq. (14), the left-hand side of Eq. (18) reads as:

$$
\kappa_{x}=\underbrace{\frac{h_{x x x}}{f_{1}^{3 / 2}}-3 \mathrm{Ca}_{\mathrm{b}}^{2 / 3} \frac{h_{x} h_{x x}^{2}}{f_{1}^{5 / 2}}}_{\text {curvature, first term }}+\underbrace{\frac{H h_{x}}{\mathrm{Ca}_{\mathrm{b}}^{2 / 3}(1-H h)^{2} f_{1}^{3 / 2}}\left[H f_{1}-\mathrm{Ca}_{\mathrm{b}}{ }^{2 / 3}(1-H h) h_{x x}\right]}_{\text {curvature, second term }},
$$


where

$$
f_{1}=1+\mathrm{Ca}_{\mathrm{b}}{ }^{2 / 3} h_{x}^{2}
$$

A detailed analysis of the importance of the various terms appearing in Eqs. (18) and (19) is included in the Supplemental Material.

The presence of a liquid upflow with average velocity $U_{l}$ does not appear explicitly in Eq. (18). However, $U_{l}$ and $U_{b}$ are linked via the material balance of the liquid flow (see Appendix B for full derivation)

$$
\mathrm{Ca}_{\mathrm{b}}=\frac{\mathrm{Ca}_{1}}{(1-H)^{2}}+\mathrm{Bo}\left[-\frac{(1-H)^{2} \log (1-H)}{2}-\frac{1}{2}+\frac{1}{8(1-H)^{2}}+\frac{3(1-H)^{2}}{8}\right],
$$

where $\mathrm{Ca}_{1} \equiv \mu U_{l} / \sigma$. The ODE can be solved for independent values of $\mathrm{Ca}_{\mathrm{b}}$ and Bo to yield the uniform film thickness $H$, then utilizing Eq. (21) to extract an estimate of $\mathrm{Ca}_{1}$. Alternatively, we assume that $\mathrm{Ca}_{1}$ is known, then Eqs. (18) and (21) can be solved iteratively to yield converged values of both $H$ and $\mathrm{Ca}_{\mathrm{b}}$.

\section{B. Numerical integration}

The profile of the front meniscus of the elongated bubble, for a given set of $\mathrm{Ca}_{\mathrm{b}}$ and Bo numbers, can be obtained by a numerical integration of Eq. (18). The numerical integration is performed by means of the Matlab solver ode45. The integration starts from the flatfilm region, point $C$ in Fig. 1, where the boundary conditions $h\left(x_{C}\right)=1, h_{x}\left(x_{C}\right)=0$ and $h_{x x}\left(x_{C}\right)=0$ apply, and proceeds towards $x \rightarrow+\infty$. The initial conditions for the numerical solution are derived as a linear perturbation of the boundary conditions above, $h(x)=1+e^{s x}, h_{x}(x)=s e^{s x}, h_{x x}(x)=s^{2} e^{s x}$, where $\delta=e^{s x}$ is a small perturbation (set to $10^{-4}$ in our calculation) and $s$ is the solution of the following equation derived by linearization of Eq. (18):

$$
s^{3}+\frac{H^{2}}{\mathrm{Ca}_{\mathrm{b}}^{2 / 3}(1-H)^{2}} s-3\left(1+T^{2}\right)=0 .
$$

The bubble cap does not end with a static meniscus region when the capillary number is increased above $10^{-3}$ (see Fig. 1(f) in the Supplemental Material), and hence the matching to a static profile as done by Bretherton [12] cannot be used. Therefore, Eq. (18) is integrated until the interface profile reaches the channel axis, point $\mathrm{A}$ in Fig. 1 , where $h=1 / H$. 
Since the nondimensional film thickness $H$ depends on both $\mathrm{Ca}_{\mathrm{b}}$ and $\mathrm{Bo}$ and is not known beforehand, the first numerical integration is performed with an initial guess for the value of $H$. Once terminated, the numerical integration is repeated by updating the value of $H$, according to an iterative procedure. This procedure has the objective to find the value of $H$ that yields an interface profile that satisfies the asymptotic condition $[15,17]$

$$
\kappa_{1}=\kappa_{2}, \quad \text { when } \quad h=\frac{1}{H}
$$

The results of validation tests conducted versus literature data for the Bo $\ll 1$ regime are reported in the Supplemental Material. The bubble profiles and uniform film thickness obtained utilizing the approximate model in the Bo $\sim 1$ regime will be compared with experimental results in Section III D.

\section{Experimental setup}

Experiments in the $\operatorname{Re}_{\mathrm{b}} \ll 1\left(\operatorname{Re}_{\mathrm{b}} \approx 10^{-2}\right)$ regime were performed using glycerol as the liquid phase (density $\rho=1186 \mathrm{~kg} / \mathrm{m}^{3}$, viscosity $\mu=0.84 \mathrm{~Pa} \cdot \mathrm{s}$ and surface tension $\sigma=$ $0.063 \mathrm{~N} / \mathrm{m}$ ) and air as the gas phase. Three different radii of glass tubes $R=1.01,1.51$ and $2.91 \mathrm{~mm}$ corresponding to $\mathrm{Bo}=0.19,0.42$ and 1.56 were tested, thus allowing investigation of bubble dynamics as the Bond number transitions from below to above the critical value of 0.842 [12]. The tube diameters were measured optically using pre-calibrated microscope objectives. The glass tubes were $30 \mathrm{~cm}$ long and were held vertical within a refractive-indexmatching box that ensured no optical deformation in the images due to the curvature of the tube wall [34]. The rigid glass capillary was connected to a syringe pump using flexible tubings to inject liquid at constant flow rate. A long air bubble was injected through a T-junction upstream of the glass capillary. This setup was used to study only the $\operatorname{Re}_{\mathrm{b}} \ll 1$ regime due to the large flow development length required in $\operatorname{Re}_{\mathrm{b}} \gg 1$ conditions.

The region of interest (ROI) was illuminated from the back using a LED light panel and shadowgraph images, see examples in Fig. 2, were captured at the rate of 30 frames per second through the front side of the refractive-index-matching box using a Nikon D5100 camera. A horizontal homemade tube microscope with $10 \mathrm{X}$ and $5 \mathrm{X}$ objectives was used for imaging the ROI on the centreplane of the glass capillaries of $R=1.01$ and $1.51 \mathrm{~mm}$, respectively, whilst a macrolens was used for the larger radius tube. These configurations 


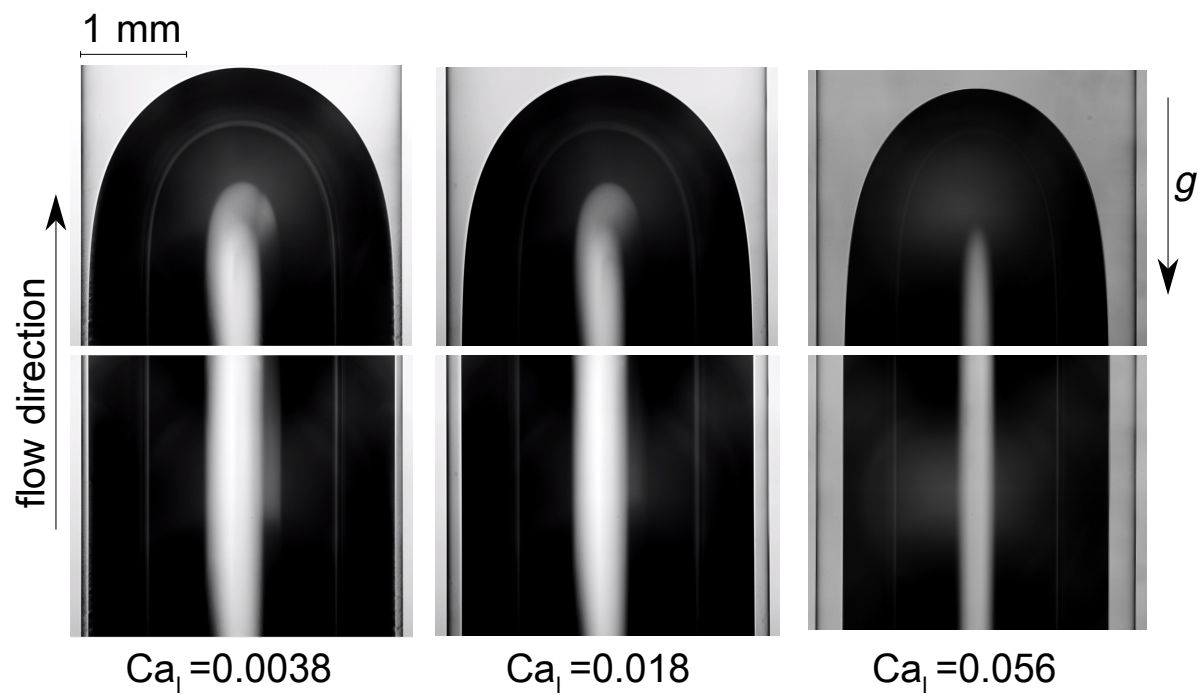

Figure 2. Examples of bubble shapes extracted from experiments in the $R=1.51 \mathrm{~mm}$ tube $(\mathrm{Bo}=$ $0.42)$ at different liquid flow rates $\left(\operatorname{Re}_{1} \ll 1\right)$. Images contain visualization of the nose and of the central part of the bubble, where the film thickness is uniform.

ensured a minimum number of 10 pixels in the film region, so that the relative error in the film thickness measurement never exceeded $10 \%$. The interface between the air bubble and the liquid phase was detected in the thresholded image sequence using a Canny edge detection algorithm [34]. The flow rate of the liquid phase, thus $\mathrm{Ca}_{1}$, was the control parameter in all experiments, whilst the corresponding $\mathrm{Ca}_{\mathrm{b}}\left(\mathrm{Ca}_{\mathrm{b}}=10^{-3}-0.15\right)$ was measured by tracking the nose of the bubble at each test condition. The experimental uncertainties for the average liquid velocity, bubble velocity, liquid viscosity and surface tension are 0.5\%,1\%,4\% and $1 \%$, respectively, that yield less than $5 \%$ uncertainty in the calculated values of $\mathrm{Ca}_{1}$ and $\mathrm{Ca}_{\mathrm{b}}$. The steady-state motion of the bubble at the measurement section was verified by calculating the velocity of the bubble nose frame-by-frame while the bubble crossed the ROI.

\section{Theoretical predictions and experimental results}

The theoretical model based on the numerical integration of Eq. (18) is utilized to obtain predictions of the profile of the bubble nose, bubble velocity and uniform film thickness under the experimental conditions. In the experiments, the liquid capillary number $\mathrm{Ca}_{1}$ is the 
control parameter, while the solution of the ODE requires knowledge of $\mathrm{Ca}_{\mathrm{b}}$. Therefore, the theoretical model is run iteratively, according to the following steps: (i) the input values of $\mathrm{Ca}_{\mathrm{l}}$ and Bo are defined; (ii) at the first iteration, $\mathrm{Ca}_{\mathrm{b}}$ is taken equal to $\mathrm{Ca}_{1}$; (iii) Equation (18) is solved iteratively, starting with a guess value of $H$, to find the value of $H$ that satisfies the asymptotic condition Eq. (23); (iv) the material balance in Eq. (21) is used, with the converged value of $H$ from the previous iteration, to update $\mathrm{Ca}_{\mathrm{b}}$; (v) steps (iii) and (iv) are repeated until the relative variation of $\mathrm{Ca}_{\mathrm{b}}$ between two consecutive iterations is below $10^{-3}$. The profile of the bubble nose, the nondimensional film thickness $H$ and bubble capillary number $\mathrm{Ca}_{\mathrm{b}}$ at convergence are the final results corresponding to $\mathrm{Ca}_{1}$ and Bo.

The shapes of the bubble nose predicted by the model and extracted from the experiments for selected values of $\mathrm{Ca}_{1}$ and Bo are presented in Fig. 3. It can be seen that as the Bond number is increased, the liquid film becomes thicker as more liquid flows downwards through the film region. A comparison of the entire experimental database with the liquid film

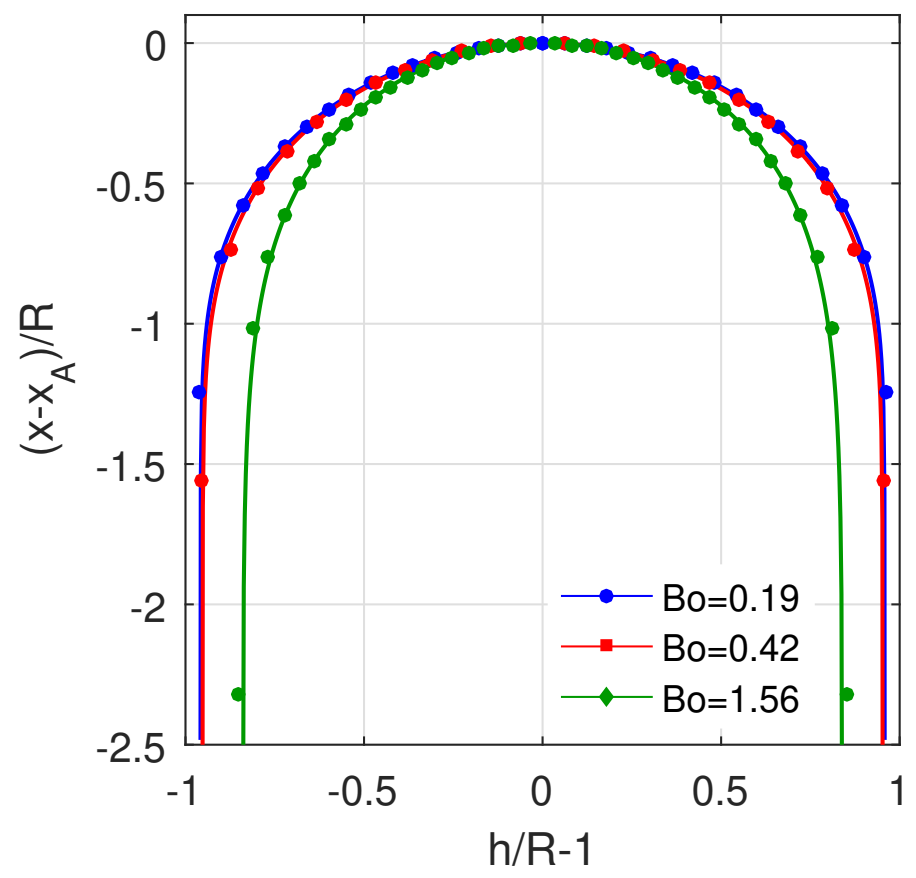

Figure 3. Comparison of shapes of the bubble front predicted by the theoretical model (solid lines) and shapes extracted from the experiments (symbols). Flow conditions all refer to $\mathrm{Ca}_{1} \approx 0.005$ $\left(\operatorname{Re}_{\mathrm{l}} \ll 1\right)$. In this figure, $x$ and $h$ indicate dimensional quantities. 


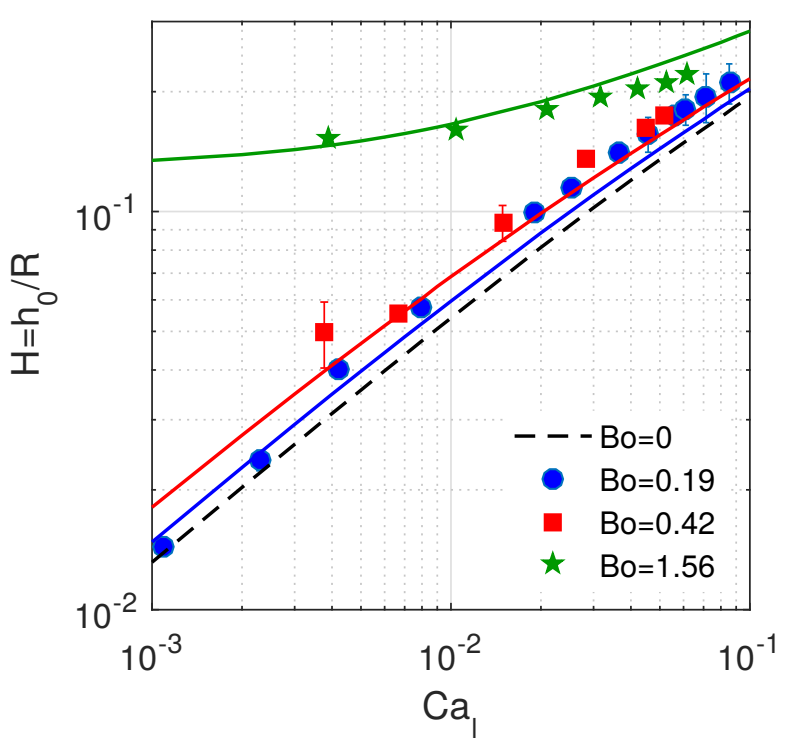

(a)

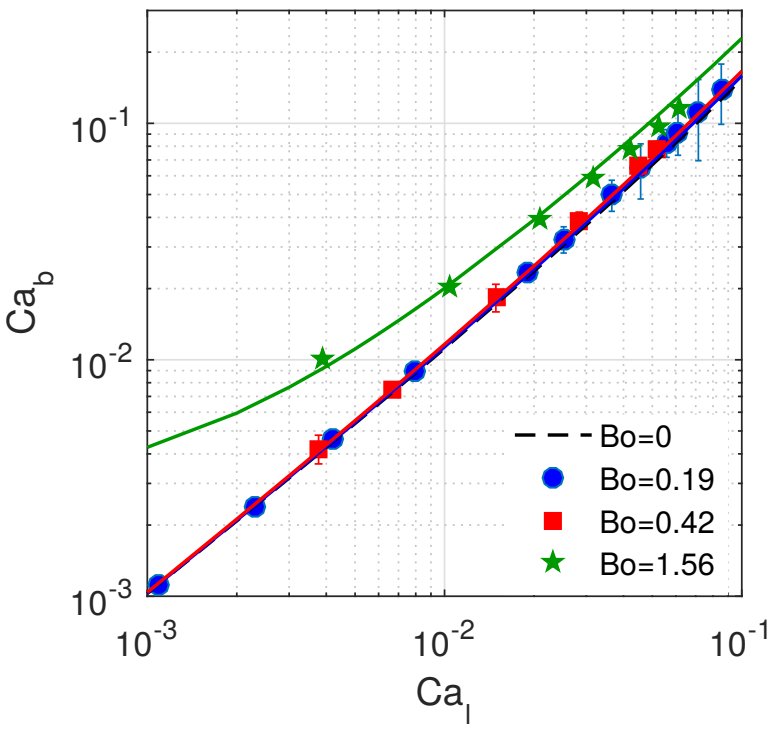

(b)

Figure 4. Comparison of the (a) thickness of the uniform film region and (b) capillary number associated to the bubble velocity given by the model (solid lines) and experiments (symbols), $\mathrm{Re}_{\mathrm{l}} \ll 1$. The error bars (in some cases obscured by the solid symbols) refer to the experimental uncertainty measured by taking the average of three measurements for each test condition.

thickness and bubble velocity obtained with the theoretical model is provided in Fig. 4. The results are displayed as a function of $\mathrm{Ca}_{1}$ because, for a given geometry, the average liquid velocity was the control parameter. The predictions for negligible gravity effects, Bo $=0$, are also reported as a reference.

When the capillary number of the liquid is increased (see Fig. 4(a)), i.e. when the average velocity of the liquid in the experiment is increased, the liquid film becomes thicker as expected from the traditional capillary theory. The nondimensional film thickness increases with the Bond number, i.e. the diameter of the tube in the experiment, and already at the lowest Bond number tested $H$ is larger than the value predicted for Bo $=0$. Hence, buoyancy influences the flow even when Bo $<0.842$. The results for different Bond numbers seem to converge at high $\mathrm{Ca}_{1}$, thus indicating that viscous forces overcome buoyancy forces. However, as the average liquid velocity $\left(\mathrm{Ca}_{1}\right)$ is reduced, both the nondimensional film thickness and bubble velocity $\left(\mathrm{Ca}_{\mathrm{b}}\right)$ exhibit very different trends depending on the Bond number. For $\mathrm{Bo}=0.19$ and $0.42, \mathrm{Ca}_{\mathrm{b}} \rightarrow 0$ as $\mathrm{Ca}_{\mathrm{l}} \rightarrow 0$ (the bubble slows down) and the film thickness 
decreases continuously following a $\mathrm{Ca}_{1}^{2 / 3}$ scaling analogous to the Bo $=0$ case (assuming that $\mathrm{Ca}_{\mathrm{b}} \approx \mathrm{Ca}_{\mathrm{l}}$ as $\left.\mathrm{Ca}_{\mathrm{b}} \rightarrow 0\right)$. Therefore, as the liquid velocity decreases, buoyancy alone is not sufficient to sustain the upward motion of the bubble, although the liquid film remains thicker than the case where gravity is negligible. In contrast, for Bo $=1.56$ as the liquid flow rate is decreased both the bubble speed and film thickness decrease down to asymptotic values. This result is in line with Bretherton's theory, for which if $\mathrm{Bo}>\mathrm{Bo}_{\mathrm{cr}}=0.842$, the bubble would still rise in the absence of a mean liquid flow.

The theoretical model captures well the experimental trends of liquid film thickness and bubble speed; the model slightly underpredicts $H$ at lower Bo and overpredicts it at larger Bo. Nonetheless, the average deviations between experimental data and theoretical results are $10 \%$ for $H$ and $5.3 \%$ for $\mathrm{Ca}_{\mathrm{b}}$, which are within the experimental uncertainties. In order to better understand the dynamics of the bubble in the Bo $\sim 1$ regime, in the next section we outline the results of a parametric analysis conducted by using the theoretical model.

\section{E. Discussion}

A parametric analysis was performed by utilizing the theoretical model to systematically explore the range $\mathrm{Ca}_{1}=10^{-6}-10^{-1}$ and $\mathrm{Bo}=0-2.5$. The predictions for the liquid film thickness as a function of $\mathrm{Ca}_{1}$ are presented in Fig. 5(a). In agreement with the trends observed in Fig. 4, the following three regimes are identified: (i) when $\mathrm{Ca}_{1}$ approaches a value of about 0.1, the trends of the liquid film thickness for different Bond numbers tend to converge as viscous forces become dominant over buoyancy effects; (ii) when $\mathrm{Ca}_{1} \rightarrow 0$ and $\mathrm{Bo}<\mathrm{Bo}_{\mathrm{cr}}$, the curves follow the same trend as the $\mathrm{Bo}=0$ case, although $H$ increases considerably with Bo; (iii) when $\mathrm{Ca}_{1} \rightarrow 0$ and $\mathrm{Bo}>\mathrm{Bo}_{\mathrm{cr}}$, the uniform film thickness (and the bubble speed, not shown here) decreases down to an asymptotic value, which is a function of Bo. The results recompiled as a function of the Bond number are illustrated in Fig. 5(b). When the liquid capillary number is decreased below 0.1, the liquid film thickness may vary by orders of magnitude as the Bond number crosses the critical value $\mathrm{Bo}_{\mathrm{cr}}=0.842$. This transition becomes steeper as the capillary number of the liquid is further reduced; the highest rate of change of $H$ versus $\mathrm{Bo}$ is always detected at $\mathrm{Bo}=\mathrm{Bo}_{\mathrm{cr}}$.

We have verified that the correction term proposed by Bretherton [12] to include buoyancy effects in his lubrication theory for bubbles displaced by a flowing liquid in a vertical tube, 


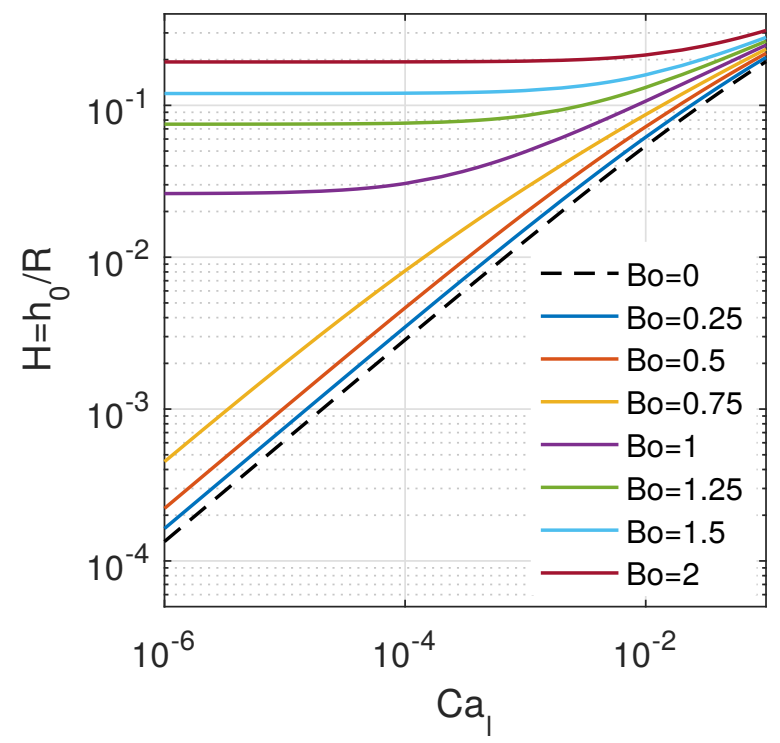

(a)

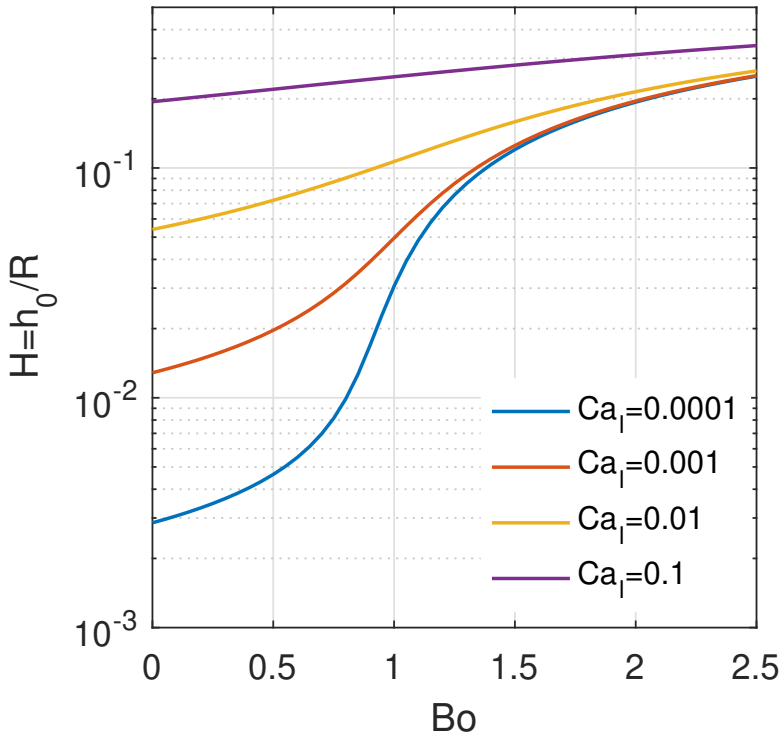

(b)

Figure 5. Predictions of the thickness of the uniform film region given by the theoretical model when varying (a) the capillary number of the liquid flow and (b) the Bond number, for $\operatorname{Re}_{1} \ll 1$.

$H(\mathrm{Bo}>0) / H(\mathrm{Bo}=0)=1+\frac{2}{3} \mathrm{Bo}$, agrees with the model's predictions in the asymptotic limit that $\mathrm{Ca}_{\mathrm{b}} \rightarrow 0$ and $\mathrm{Bo} \rightarrow 0$. Deviations are below $10 \%$ when $\mathrm{Ca}_{\mathrm{b}} \lesssim 10^{-3}$ and $\mathrm{Bo} \lesssim 0.4$.

A further analysis of the transition value of the Bond number was performed by running the theoretical model with a very small value of the liquid capillary number, $\mathrm{Ca}_{1}=10^{-10}$, to simulate the limit of no imposed liquid flow. The results are reported in Fig. 6. The predictions of the present model confirm the critical value derived by Bretherton [12], as the values of the bubble capillary number sharply deviate from the Bo $=0$ case (where $\mathrm{Ca}_{\mathrm{b}} \approx \mathrm{Ca}_{\mathrm{l}}=10^{-10}$ ) as the Bond number is increased above the value 0.842. Figure 6 includes also a curve depicting the capillary number of the bubble estimated using a relationship derived in the second part of Bretherton's paper:

$$
\text { Bo }-0.842=1.25 \mathrm{Ca}_{\mathrm{b}}^{2 / 9}+2.24 \mathrm{Ca}_{\mathrm{b}}^{1 / 3} \text {. }
$$

Equation (24) agrees remarkably well with the results of the present model for $0.842<$ Bo $\lesssim 1$, while deviations quickly grow above $10 \%$ when Bo is increased above 1 . As pointed out by Bretherton, the deviations may be attributed to his assumption that the interface curvature due to the axial symmetry, $\kappa_{2}$, is constant along the thin-film. 


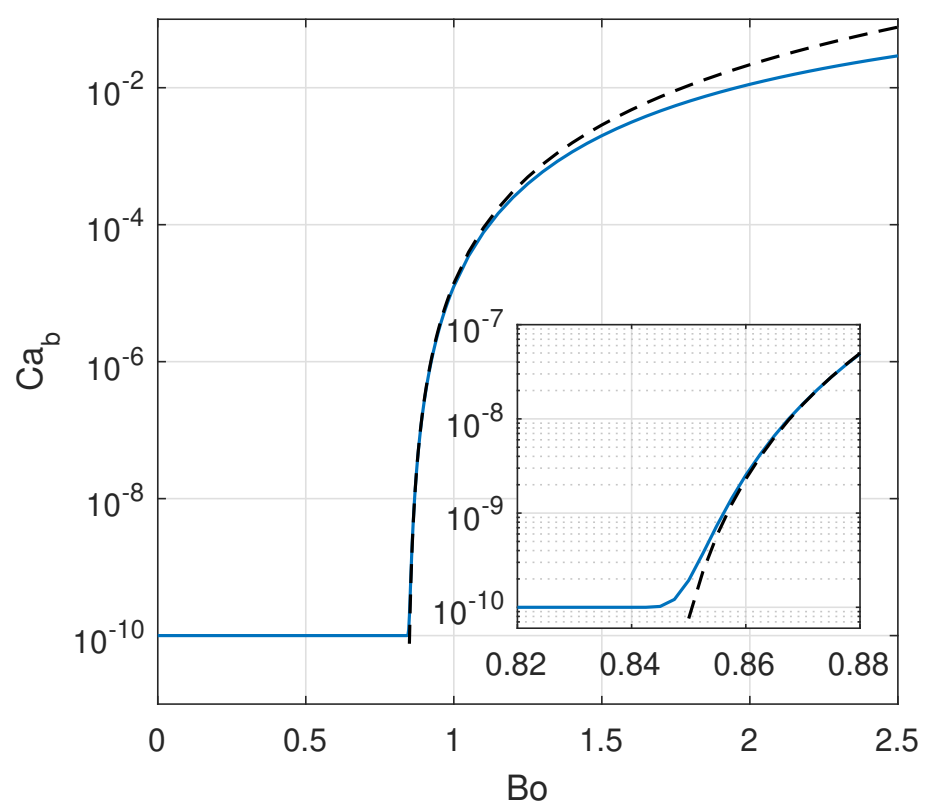

Figure 6. Trends of the bubble capillary number versus Bond number predicted by the model (blue line) when the liquid capillary number is set to a very small value, $\mathrm{Ca}_{1}=10^{-10}$ (and $\mathrm{Re}_{\mathrm{l}} \ll 1$ ). The black dashed line indicates the results of the theory developed by Bretherton [12] for long bubbles rising in a stagnant liquid, Eq. (24).

Following the approach of Aussillous and Quéré [14], a scaling analysis for the liquid film thickness in the $\mathrm{Bo}<\mathrm{Bo}_{\mathrm{cr}}$ regime is developed $\left(\mathrm{Re}_{\mathrm{b}} \ll 1\right)$. Balancing the viscous force with the pressure gradient and buoyancy term in the equation of motion along the dynamic meniscus at the bubble front yields

$$
\frac{\mu U_{b}}{h_{0}^{2}} \approx \frac{1}{\ell} \frac{\sigma}{R-h_{0}}+\rho g
$$

where the characteristic length of the dynamic meniscus $\ell$ can be derived by matching the curvatures of the dynamic and static menisci,

$$
\frac{h_{0}}{\ell^{2}}+\frac{1}{R-h_{0}} \approx \frac{2}{R-h_{0}}-\frac{1}{a}
$$

with $a=\sqrt{\sigma /(\rho g)}$ being the capillary length scale. Equation (26) is expected to hold as long as buoyancy effects are mild ( $\left.\mathrm{Bo} \lesssim \mathrm{Bo}_{\mathrm{cr}}\right)$, because the curvature of the static menisci is calculated as the curvature of the bubble nose in the absence of gravity effects, $2 /\left(R-h_{0}\right)$, 
with the correction $-1 / a$, which accounts for the linear curvature reduction induced by buoyancy (see Fig. 1(d) in the Supplemental Material). Using Eq. (26) to extract $\ell$ and substituting it into Eq. (25), an implicit scaling law for the nondimensional film thickness is derived:

$$
H \approx \frac{\mathrm{Ca}_{\mathrm{b}}{ }^{2 / 3}}{\mathrm{Ca}_{\mathrm{b}}{ }^{2 / 3}+\left[\sqrt{1-\left(\mathrm{Bo}^{\prime}\right)^{1 / 2}}+\mathrm{Bo}^{\prime}\left(\frac{H}{1-H}\right)^{1 / 2}\right]^{2 / 3}},
$$

where $\mathrm{Bo}^{\prime} \equiv \rho g\left(R-h_{0}\right)^{2} / \sigma$. Using Aussillous and Quéré's [14] scaling for the film thickness at large capillary numbers to express the term $H /(1-H)$ at the denominator of Eq. (27), the following scaling law is finally obtained:

$$
H \approx \frac{\mathrm{Ca}_{\mathrm{b}}{ }^{2 / 3}}{\mathrm{Ca}_{\mathrm{b}}{ }^{2 / 3}+\left[\sqrt{1-\left(\mathrm{Bo}^{\prime}\right)^{1 / 2}}+\mathrm{Bo}^{\prime} \mathrm{Ca}_{\mathrm{b}}{ }^{1 / 3}\right]^{2 / 3}} .
$$

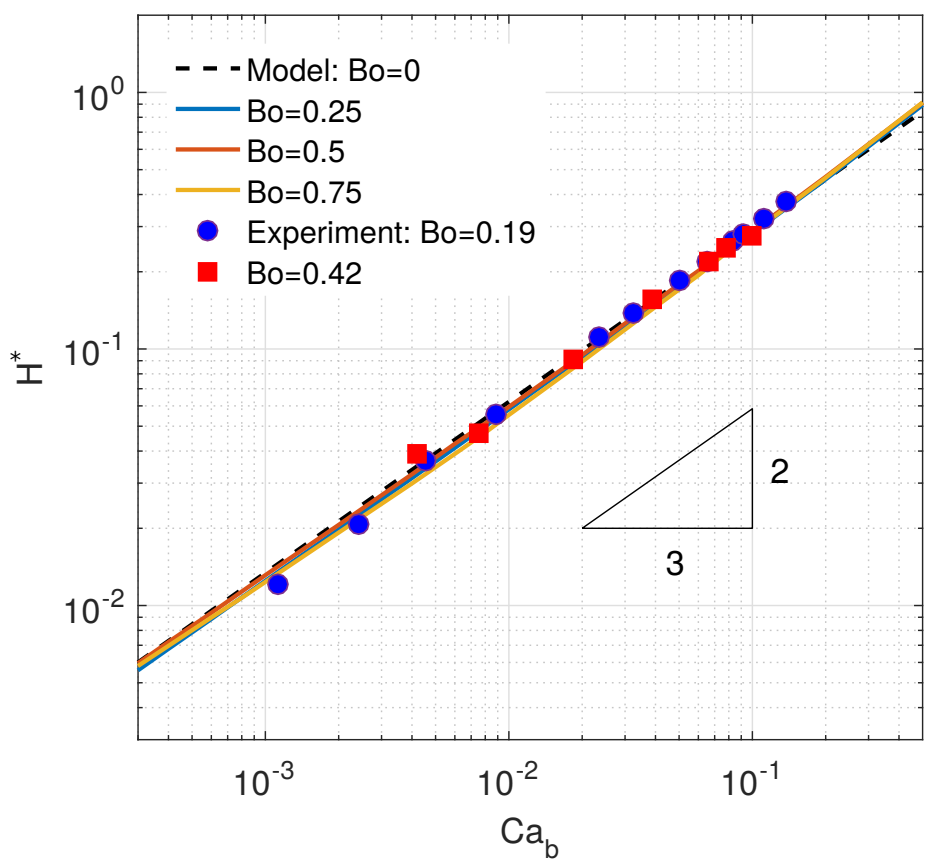

Figure 7. Scaling performance of Eq. (28) versus the theoretical and experimental data $\left(\operatorname{Re}_{1} \ll 1\right)$ for $\mathrm{Bo}<\mathrm{Bo}_{\mathrm{cr}}$. The rescaled film thickness $H^{*}$ is defined as $H^{*}=$ $H\left[3.35 \mathrm{Ca}_{\mathrm{b}}{ }^{2 / 3}+\left(\sqrt{1-1.12 \mathrm{Bo}^{1 / 2}}+\sqrt{1.34} \mathrm{Bo} \mathrm{Ca}_{\mathrm{b}}{ }^{1 / 3}\right)^{2 / 3}\right]$. The rescaled data collapse along a $\mathrm{Ca}_{\mathrm{b}}{ }^{2 / 3}$ line. 
The entire experimental and theoretical film thickness database for $\mathrm{Bo}<\mathrm{Bo}_{\mathrm{cr}}$, rescaled as indicated in Eq. (28), is plotted in Fig. 7. For simplicity, it is assumed that $\mathrm{Bo}^{\prime} \approx \mathrm{Bo}$. The

rescaled data collapse along a $\mathrm{Ca}_{\mathrm{b}}{ }^{2 / 3}$ line. The coefficients 3.35 and $\sqrt{1.34}$ used to rescale the data in Fig. 7 derive from Aussillous and Quéré's [14] fit of Taylor's data and Bretherton's law, respectively, while the coefficient 1.12 is obtained by a best fit of the present database.

\section{FLOWS WITH INERTIA}

In this section, we investigate the dynamics of the bubble in the $\operatorname{Re}_{\mathrm{b}} \gg 1$ regime. The theoretical model including inertial effects is developed in Sections IV A and B; the numerical model for the VOF-based solution of the full Navier-Stokes equations is described in Sec. IV C; the results obtained with the theoretical model and the full numerical simulations are compared and discussed in Sections IV D and E.

\section{A. Approximate model}

Inertial forces are introduced in the theoretical model based on Eqs. (3)-(7) with the assumption that $\operatorname{Re}_{\mathrm{b}}=\mathcal{O}\left(\epsilon^{-3}\right)$, so that the inertial term in the $x$-momentum equation is of the same order as the pressure gradient while that in the $y$-momentum equation is negligible. Hence, the nondimensional $x$-momentum equation at leading-order in $\epsilon$ becomes

$$
\frac{1}{2} \mathrm{Ca}_{\mathrm{b}}{ }^{1 / 3} \operatorname{Re}_{\mathrm{b}} H\left(u u_{x}+v u_{y}\right)=-p_{x}+u_{y y}-T^{2}
$$

while the other equations governing the flow at leading-order, Eqs. (10a) and (10c), and the boundary conditions, Eqs. (5), (12) and (13), remain unchanged.

Due to the presence of the nonlinear inertial term on the left-hand side of Eq. (29), the procedure to derive the ODE governing the film profile differs from the $\mathrm{Re}_{\mathrm{b}} \ll 1$ case. We adopt the methodology originally introduced by Shkadov [35] to study the nonlinear dynamics of waves appearing on the surface of a thin liquid film flowing along a vertical 
plate. The continuity and $x$-momentum equations are integrated from $y=0$ to $y=h(x)$ :

$$
\begin{aligned}
\int_{0}^{h} u_{x} d y+\int_{0}^{h} v_{y} d y & =0 \\
\frac{1}{2} \mathrm{Ca}_{\mathrm{b}}{ }^{1 / 3} \operatorname{Re}_{\mathrm{b}} H\left(\int_{0}^{h} u u_{x} d y+\int_{0}^{h} v u_{y} d y\right) & =\int_{0}^{h} \kappa_{x} d y+\int_{0}^{h} u_{y y} d y-\int_{0}^{h} T^{2} d y,
\end{aligned}
$$

where $p_{x}=-\kappa_{x}$ has been used. In order to evaluate the integrals appearing in the equations above, a closure relationship for the velocity profile in the liquid film $u(x, y)$ is necessary. This profile must satisfy the boundary conditions $u=-1$ at $y=0$ and $u_{y}=0$ at $y=h(x)$, and continuity as expressed in Eq. (16), and therefore the following parabolic profile is chosen:

$$
u(x, y)=\frac{3}{h^{3}}\left(\frac{T^{2}}{3}+1-h\right)\left(\frac{y^{2}}{2}-h y\right)-1 .
$$

Using Eq. (31) to express $u$ and its derivatives, and the continuity Eq. (30a) to derive a relationship for $v$, Eq. (30b) yields a new third-order ODE for the liquid film profile in the presence of inertial effects:

$$
\kappa_{x}=\underbrace{3 \frac{(h-1)}{h^{3}}}_{\text {viscous term }}+\underbrace{T^{2} \frac{\left(h^{3}-1\right)}{h^{3}}}_{\text {buoyancy term }}+\underbrace{\frac{1}{10} H \mathrm{Ca}_{\mathrm{b}}^{1 / 3} \operatorname{Re}_{\mathrm{b}}\left(h^{2}-6-4 T^{2}-\frac{2}{3} T^{4}\right) \frac{h_{x}}{h^{3}}}_{\text {inertial term }},
$$

while $\kappa_{x}$ can be expressed as indicated in Eq. (19). A detailed analysis of the importance of the various terms appearing in Eq. (32) is included in the Supplemental Material.

\section{B. Numerical integration}

The numerical integration of Eq. (32) is performed as for the $\mathrm{Re}_{\mathrm{b}} \ll 1$ case, see Section III B. The linearized conditions to start the integration procedure, appropriate to the uniform film region, are now extracted from the solution of the equation:

$$
s^{3}+\left[\frac{H^{2}}{\mathrm{Ca}_{\mathrm{b}}^{2 / 3}(1-H)^{2}}+\frac{1}{10} H \mathrm{Ca}_{\mathrm{b}}^{1 / 3} \operatorname{Re}_{\mathrm{b}}\left(5+4 T^{2}+\frac{2}{3} T^{4}\right)\right] s-3\left(1+T^{2}\right)=0 .
$$

The numerical integration for the front meniscus starts from the flat-film region and proceeds towards $x \rightarrow+\infty$. The iterative procedure adopted to fix the value of $H$ is the same as explained in Section III B. 
Inertial effects have been demonstrated to yield significant undulations on the surface of the elongated bubble [17], near its rear meniscus. Therefore, the combined effect of buoyancy and inertial forces on the profile of the rear meniscus is of interest. The profile of the rear meniscus of the bubble in the presence of inertia can be calculated by numerical integration of Eq. (32), starting from close to the flat-film region (point $D$ in Fig. 1) and proceeding towards $x \rightarrow-\infty$. The nondimensional film thickness $H$ is now fixed by the solution at the front meniscus. As discussed by Magnini et al. [17], the linearized initial condition for the solution at the bubble back is a sinusoidal function that contains a phase shift $\varphi$ as an integration constant. Different values of $\varphi$ yield interface profiles that are shifted along $x$. Therefore, an iterative procedure is utilized to find the value of $\varphi$ which gives a profile of the rear meniscus that satisfies the asymptotic condition reported in Eq. (23). Profiles of the bubble rear obtained by numerical integration will be compared with the results of full numerical simulations in Section IV D.

\section{Numerical simulations}

Numerical simulations of the co-current flow of liquid and an elongated bubble in the $\mathrm{Re}_{\mathrm{b}} \gg 1$ regime were performed by means of the open-source CFD package ESI OpenFOAM, release 2.3.1. The unsteady Navier-Stokes equations are solved for both the liquid and gas phases, which are treated as a single mixture fluid according to a Volume-Of-Fluid (VOF) [31] formulation. The flow is assumed incompressible and the fluid Newtonian. The surface tension force is included within the momentum equation as a body force according to the Continuum Surface Force (CSF) method [36]. The self-developed flexCLV (flexible coupled Level Set and VOF) [37] algorithm is adopted to enhance the calculation of the surface tension force. This routine reconstructs a level set function (distance from the liquid-gas interface) from the VOF volume fraction field at every time-step, and then evaluates the interface topology (curvature and normal vector) based on derivatives of the level set.

The numerical simulations are run with an axisymmetric flow model. An elongated bubble is initialized near the inlet boundary of the domain (as in Fig. 1). The initial bubble length is of about $20 R$, in order to ensure that a flat-film region is formed between the front and rear caps. At the inlet boundary, liquid enters the domain with a parabolic velocity profile. A no-slip condition is set at the wall. At the outflow section, the pressure is set to a 
zero reference value while the velocity gradient along the stream direction is set to zero [38]. The gravitational force acts parallel to the axis of the tube and is directed towards the inlet boundary. The liquid to gas density and viscosity ratios are respectively fixed to 1000 and 100. These ratios ensure that the results are independent of the bubble density and viscosity as indicated by the numerical study of Kang et al. [39]. The simulations are run forward in time until the bubble translates with a constant velocity. The independent parameters in the numerical model are $\mathrm{Ca}_{1}, \mathrm{Re}_{\mathrm{l}}$ and Bo. A parametric analysis is conducted by varying these groups within the range $\mathrm{Ca}_{1}=0.00464-0.069, \mathrm{Re}_{\mathrm{l}}=0.07-1400$ and $\mathrm{Bo}=0-5$.

\section{Theoretical predictions and results of numerical simulations}

The theoretical model based on the numerical integration of Eq. (32) is utilized to obtain predictions of the profiles of the bubble nose and rear, bubble velocity and uniform film thickness under the range of conditions explored by the numerical simulations. The Bond number and the liquid capillary and Reynolds numbers are the independent parameters. For each set of $\mathrm{Bo}, \mathrm{Ca}_{\mathrm{l}}$ and $\mathrm{Re}_{\mathrm{l}}$, the model is run iteratively until converged values of $H, \mathrm{Ca}_{\mathrm{b}}$ and $\mathrm{Re}_{\mathrm{b}}$ are achieved, as was done for the $\mathrm{Re}_{\mathrm{b}} \ll 1$ case (see Section IIID).

Examples of the profiles of the rear meniscus obtained with the theoretical model and the full simulations for fixed values of $\mathrm{Ca}_{1}$ and $\mathrm{Re}_{1}$ and increasing values of Bo are illustrated in Fig. 8. When $\mathrm{Bo}=0$, the undulation at the bubble rear exhibits only one crest, which suggests that inertial effects are weak [17]. This response is consistent with the Weber number of the flow being smaller than 1 , as $\mathrm{We}_{\mathrm{b}} \equiv \mathrm{Ca}_{\mathrm{b}} \mathrm{Re}_{\mathrm{b}}=0.5$. As the Bond number is increased, a significant increase of the amplitude of the undulation and a slight decrease of its wavelength are evident, with three crests becoming apparent near the bubble tail. The theoretical model based on the solution of Eq. (32) predicts very well the value of the uniform film thickness and the entire profile of rear meniscus and undulations for the three cases shown in Fig. 8, with a slight tendency to overestimate the undulation amplitude at larger Bond numbers.

A systematic analysis of the trends of the nondimensional film thickness $H$, bubble to liquid speed ratio $U_{b} / U_{l}$ and nondimensional wavelength $\lambda / \ell$ (see Fig. 1) of the interface undulation at the rear meniscus (with $\ell=h_{0} \mathrm{Ca}_{\mathrm{b}}{ }^{-1 / 3}$ ) has been performed by varying $\mathrm{Re}_{\mathrm{l}}$ and Bo, for three constant values of $\mathrm{Ca}_{1}$. The results for both the numerical simulations and 


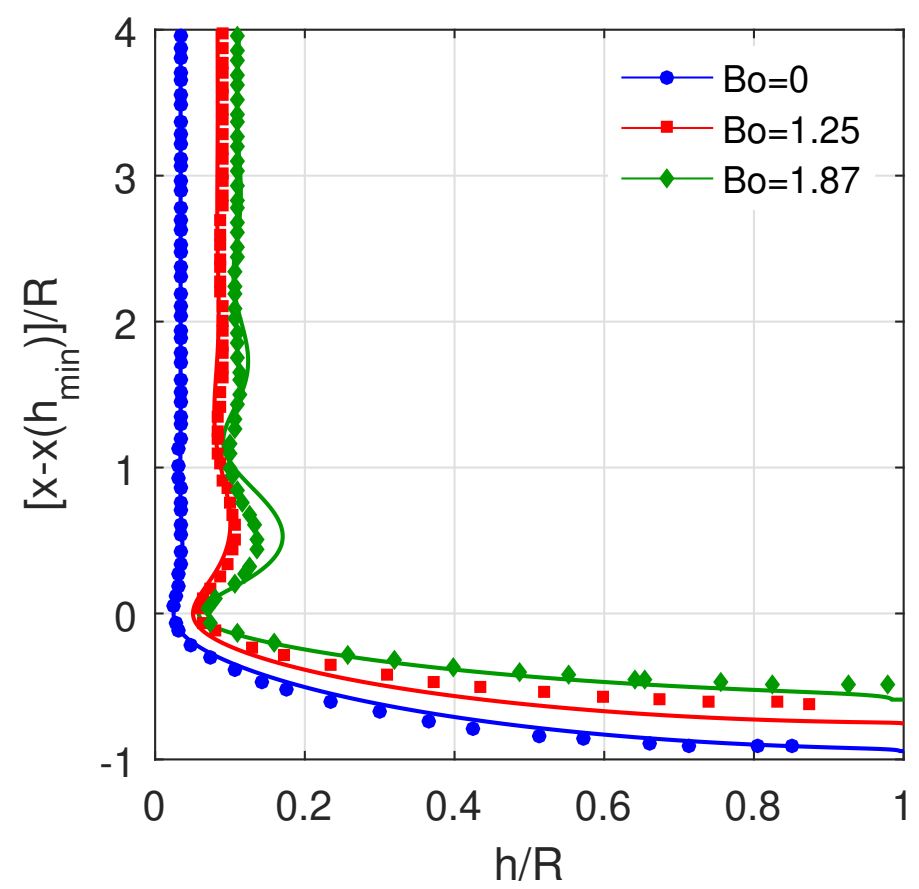

Figure 8. Comparison of shapes of the bubble rear predicted as solution of Eq. (32) (solid lines) and shapes extracted from the full numerical simulations (symbols). Flow conditions are: $\mathrm{Ca}_{1}=0.00464$ and $\mathrm{Re}_{\mathrm{l}}=92.8 . x\left(h_{\min }\right)$ denotes the axial location where the minimum value of the film thickness is measured. In this figure, $x$ and $h$ indicate dimensional quantities.

the theoretical model are summarized in Fig. 9, where they are compiled as a function of $\mathrm{Re}_{\mathrm{l}}$, which is an independent parameter in the analysis. From the numerical simulations and theoretical profiles, the wavelength of the undulation is calculated as the distance between the two most upstream crests; see the sketch in Fig. 1.

As a general trend, the liquid film thickness and speed ratio increase with the Bond number. Relative to the case of negligible gravity, Bo $=0$, buoyancy effects are more apparent at smaller capillary numbers, which is consistent with the buoyancy term in Eq. (32) being proportional to $\mathrm{Bo} / \mathrm{Ca}_{\mathrm{b}}$. The reduction of $H$ with increasing $\mathrm{Re}_{\mathrm{b}}$ at intermediate values of the Reynolds number becomes more pronounced as Bo is increased; for instance, when $\mathrm{Bo}=1.25$ and $\mathrm{Ca}_{\mathrm{l}}=0.069$ (see the orange triangles in Fig. $9(\mathrm{~g})$ ), $H$ decreases by more than $20 \%$ within the range $\operatorname{Re}_{1}=10^{-1}-10^{2}$. The velocity ratio follows the same trends versus $\mathrm{Re}_{\mathrm{l}}$ and Bo as the nondimensional film thickness.

Magnini et al. [17] showed that in the $\mathrm{Bo}=0$ case the wavelength of the undulations on 

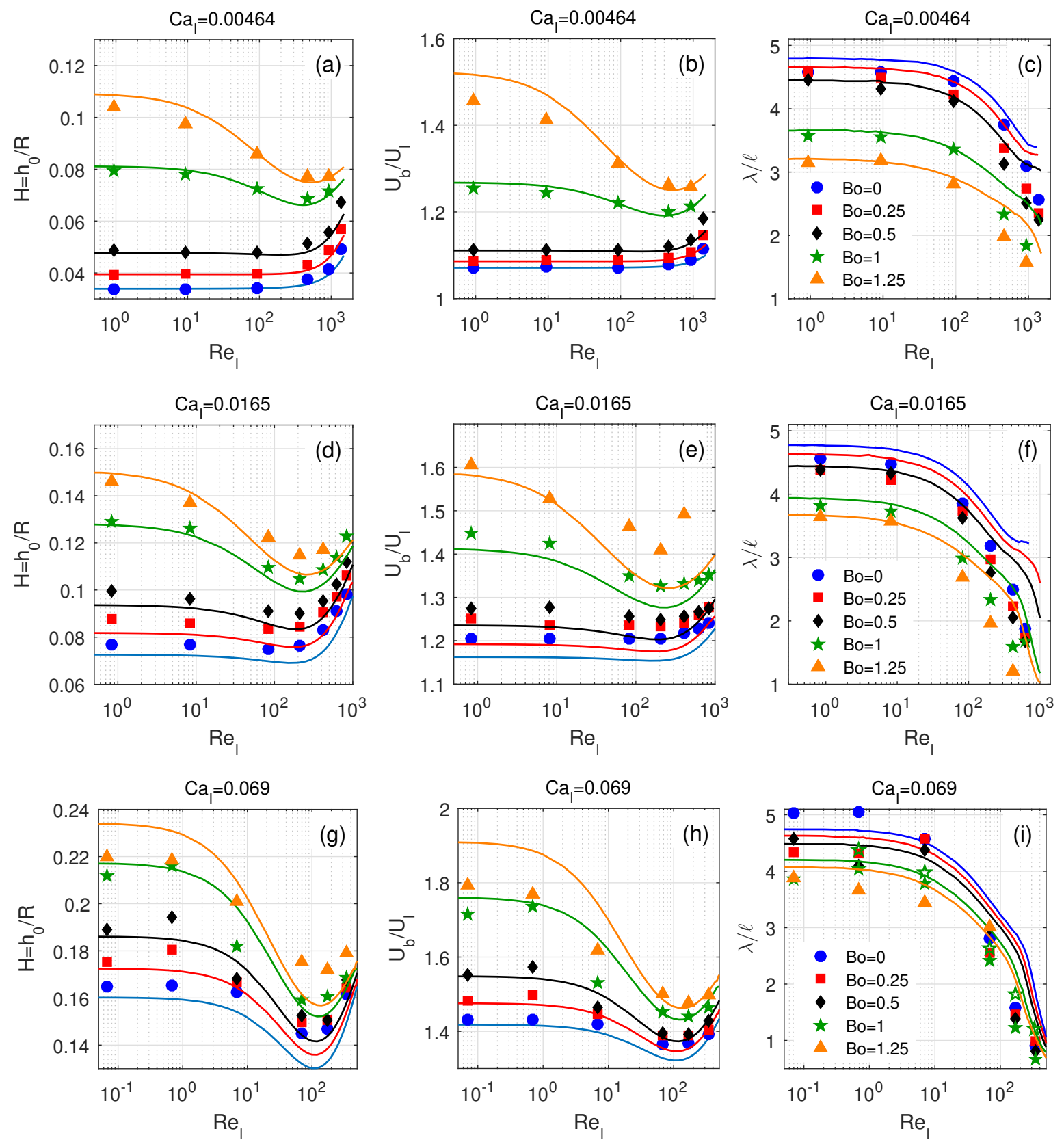

Figure 9. Comparison of the thickness of the uniform film region $H$, bubble to average liquid velocity $U_{b} / U_{l}$ and wavelength $\lambda / \ell$ of the ripples appearing at the rear meniscus of the bubble, for three constant values of the liquid capillary number. Full markers identify the results of numerical simulations and solid lines are the predictions of the model, Eq. (32).

the surface of the bubble decreases as $\mathrm{We}_{\mathrm{b}}$ increases above 0.1 due to the effects of inertial forces, while it reaches an asymptotic value $\lambda / \ell \approx 4.8-5$ (approximately independent of 
$\left.\mathrm{Ca}_{\mathrm{b}}\right)$ as $\mathrm{Re}_{\mathrm{b}} \rightarrow 0$. The present analysis demonstrates that buoyancy effects have a significant impact on the dynamics of the interfacial ripples. When inertial forces are negligible $\left(\operatorname{Re}_{1} \lesssim 1\right.$ in Figs. 9(c), (f) and (i)), the asymptotic value of the nondimensional wavelength decreases as Bo is increased. However, no appreciable change of the amplitude of the ripple is observed when increasing Bo from 0 to 1.25, so that only one undulation crest is clearly visible. When inertial forces are important $\left(\operatorname{Re}_{1} \gtrsim 10-100\right)$, gravitational effects further reduce the wavelength of the interfacial ripple and we observe that the amplitude of the undulation grows considerably. This feature cannot be attributed solely to the larger bubble velocity (i.e. larger Weber number, which increases the amplitude of the ripple and decreases its wavelength), but it represents a direct effect of buoyancy on the dynamics of the undulation.

The results of a systematic analysis of the influence of the Bond number in the range Bo $=0-5$, while keeping constant $\mathrm{Ca}_{1}$ and $\mathrm{Re}_{\mathrm{l}}$, are reported in Fig. 10. The effect of buoyancy on the flow is substantial, as the film thickens by a factor of about 4 between $\mathrm{Bo}=0$ and $\mathrm{Bo}=5$. The bubble velocity increases mildly with $\mathrm{Bo}$ as long as $\mathrm{Bo}<\mathrm{Bo}_{\mathrm{cr}}$ and more steeply at larger values of the Bond number. The wavelength of the undulation at the bubble rear diminishes considerably as gravitational forces become important, see Fig. 10(c),

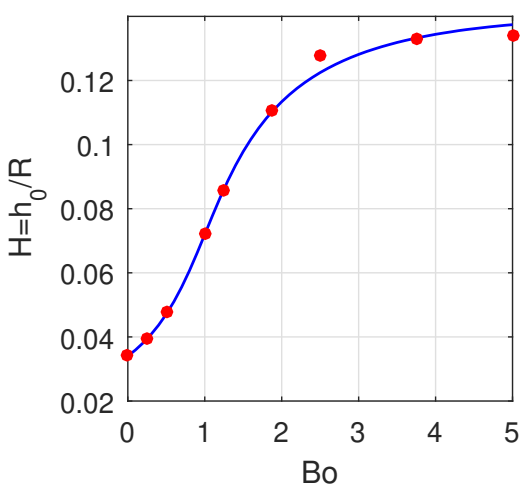

(a)

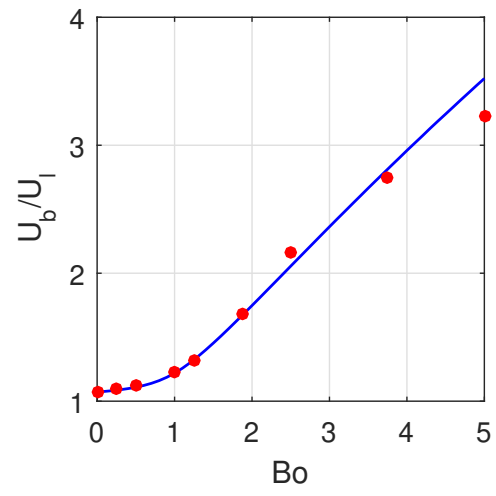

(b)

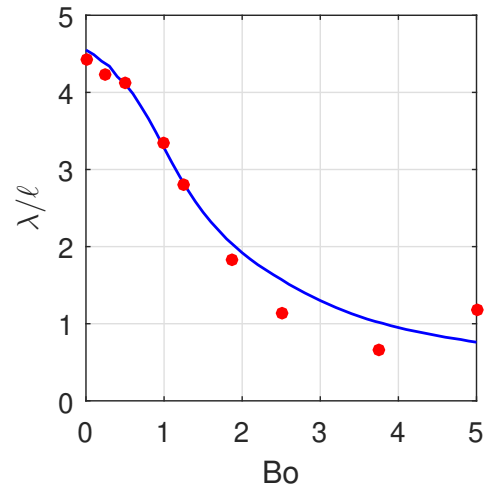

(c)

Figure 10. Comparison of the (a) thickness of the uniform film region $H$, (b) bubble to average liquid velocity $U_{b} / U_{l}$ and (c) wavelength $\lambda / \ell$ of the ripples appearing at the rear meniscus of the bubble obtained as solution of Eq. (32) (solid lines) and numerical simulations (symbols), for $\mathrm{Ca}_{\mathrm{l}}=0.00464$ and $\mathrm{Re}_{\mathrm{l}}=92.8$. The numerical simulation for $\mathrm{Bo}=5$ gave time-dependent profiles of the bubble rear, the value of the wavelength reported in the figure is an average in time. 
while the amplitude of the undulation grows as shown in Figure 8. In the range Bo $=0-5$, the Weber number of the bubble increases from about 0.5 to 5 . The data of Magnini et al. [17] for $\mathrm{Bo}=0$ and similar capillary numbers indicate that, in the same range of Weber numbers (i.e. inertial effects of comparable magnitude), the nondimensional wavelength of the ripple decreases from about $\lambda / \ell=4.5$ to 3.5 , which is a substantially smaller variation than that observed in Fig. 10(c). This further confirms that gravitational forces directly impact the profile of the undulation at the rear meniscus of the bubble.

The average deviation between the results of the numerical simulations and theoretical model for all the data points included in Figs. 9 and 10 is $4.8 \%$ for the film thickness, $2.2 \%$ for the bubble velocity and $15.3 \%$ for the undulation wavelength.

\section{E. Discussion}

The scaling analysis for the film thickness in the negligible inertia regime proposed in Section III E is now corrected to account for inertial effects. Inertial forces tend to increase the curvature of the bubble nose $[16,40]$, which is here accounted for by introducing a correction to the curvature matching condition Eq. (26):

$$
\frac{h_{0}}{\ell^{2}}+\frac{1+f_{k}}{R-h_{0}} \approx \frac{2\left(1+f_{k}\right)}{R-h_{0}}-\frac{1}{a},
$$

where $f_{k}$ can be estimated as $f_{k} \approx \mathrm{Ca}_{\mathrm{b}} \mathrm{Re}_{\mathrm{b}}^{0.9}$ according to the empirical fit of Han and Shikazono [16] to their experimental film thickness data. Balancing viscous, inertial and gravitational forces and the pressure gradient in the equation of motion along the dynamic meniscus yields

$$
\frac{\mu U_{b}}{h_{0}^{2}} \approx \frac{1}{\ell} \frac{\sigma\left(1+f_{k}\right)}{R-h_{0}}+\rho g-\frac{1}{\ell} \rho U_{b}^{2} .
$$

Using Eq. (34) to express $\ell$ in the equation above, the following scaling law is obtained:

$$
H \approx \frac{\mathrm{Ca}_{\mathrm{b}}{ }^{2 / 3}}{\mathrm{Ca}_{\mathrm{b}}{ }^{2 / 3}+\left[\left(1+f_{k}-\mathrm{We}_{\mathrm{b}}^{\prime}\right) \sqrt{1+f_{k}-\left(\mathrm{Bo}^{\prime}\right)^{1 / 2}}+\mathrm{Bo}^{\prime} \mathrm{Ca}_{\mathrm{b}}{ }^{1 / 3}\right]^{2 / 3}},
$$

where $\mathrm{We}_{\mathrm{b}}^{\prime} \equiv \rho U_{b}^{2}\left(R-h_{0}\right) / \sigma$. Equation (36) can be used to infer the influence of capillary, viscous, inertial and buoyancy forces on the uniform liquid film thickness trends presented in Figs. 9 and 10. For simplicity, we assume that $\mathrm{Bo}^{\prime} \approx \mathrm{Bo}$ and $\mathrm{We}_{\mathrm{b}}^{\prime} \approx \mathrm{We}_{\mathrm{b}}$. For small 
values of the capillary number, the positive term $\mathrm{Bo} \mathrm{Ca}_{b}{ }^{1 / 3}$ is small and the thickening of the liquid film due to buoyancy (relative to the Bo $=0$ case) is more apparent. As the capillary number is increased, the relative impact of the Bond number is reduced due to the presence of the positive $\mathrm{Ca}_{\mathrm{b}}{ }^{2 / 3}$ term in the denominator.

When $\operatorname{Re}_{\mathrm{b}} \lesssim 1$, inertial forces are negligible; $\mathrm{We}_{\mathrm{b}} \ll 1$ and $f_{k} \ll 1$ so that Eq. (36) reduces to Eq. (28). For increasing values of $\mathrm{Re}_{\mathrm{b}}, f_{k}$ increases and the argument of the square root becomes less sensitive to Bo; therefore, the values of $H$ for different Bo numbers tend to get closer, as observed in particular in Fig. $9(\mathrm{~g})$ at $\operatorname{Re}_{1} \approx 100$. This mixed effect of inertia and buoyancy forces makes the decreasing trend of $H$ with increasing $\operatorname{Re}_{1}$ more evident for larger Bo numbers, as demonstrated in the previous section. As $\mathrm{Re}_{\mathrm{b}}$ is increased

further, $f_{k} \gg \mathrm{Bo}^{1 / 2}$ and inertial effects overcome buoyancy; eventually, at large $\mathrm{Re}_{\mathrm{b}}$ the film thickness curves for different Bond numbers tend to converge as indicated in Fig. 9.

\section{CONCLUSIONS}

In this paper we performed a systematic analysis of the effect of buoyancy on the dynamics of long gas bubbles rising in a vertical circular channel in a co-current liquid flow. The bubble speed, shape, uniform film thickness and features of the undulation appearing nearby the rear meniscus have been quantified for a range of capillary numbers $\mathrm{Ca}_{\mathrm{b}}=10^{-3}-10^{-1}$, Reynolds numbers from $\operatorname{Re}_{\mathrm{b}} \ll 1$ to about $10^{3}$, and Bond numbers Bo $=0-5$. We carried out experiments to explore the $\mathrm{Re}_{\mathrm{b}} \ll 1$ regime and numerical simulations to study the $\mathrm{Re}_{\mathrm{b}} \gg 1$ regime. A theoretical model that implements inertial and buoyancy effects to extend the classical Bretherton theory was developed and utilized to run parametric analyses across the entire range of dimensionless parameters of interest. In general, when bubbles rise in a co-current liquid flow buoyancy effects are manifest already when $\mathrm{Bo}<\mathrm{Bo}_{\mathrm{cr}}=0.842$, with a substantial increase of the film thickness compared to the Bo $=0$ case. As the capillary number of the liquid tends to zero, the bubble velocity and uniform film thickness decrease indefinitely when $\mathrm{Bo}<\mathrm{Bo}_{\mathrm{cr}}$, whereas they decrease down to non-zero asymptotic values when $\mathrm{Bo}>\mathrm{Bo}_{\mathrm{cr}}$. As a consequence, when $\mathrm{Ca}_{1} \leq 10^{-3}$ small variations of the Bond number across the critical value may induce orders of magnitude changes in the liquid film thickness and bubble velocity. In the $\mathrm{Re}_{\mathrm{b}} \gg 1$ regime, the reduction of the liquid film thickness and bubble velocity observed for increasing values of the Reynolds number (up 
to $\left.\mathrm{Re}_{\mathrm{b}}=100-500\right)$ is largely amplified when $\mathrm{Bo}>0$. The undulation at the rear end of the bubble exhibits higher amplitude and smaller wavelength as the Bond number is increased. The theoretical model developed in this work quantitatively predicts the present experimental and numerical database within a $10 \%$ error with respect to the uniform film thickness and $5 \%$ for the bubble velocity.

\section{SUPPLEMENTAL MATERIAL}

Supplemental Material associated with this article includes a detailed analysis of the forces acting along the front meniscus of the bubble and the validation of the theoretical model versus literature data in the case of negligible buoyancy effects.

\section{Appendix A: Equations governing the flow in the liquid film}

The equations governing the flow in the liquid film in the axisymmetric, cylindrical coordinates introduced in Fig. 1, in dimensional form are

$$
\begin{aligned}
u_{x}+v_{y}-\frac{v}{R-y} & =0, \\
\rho\left(u u_{x}+v u_{y}\right) & =-p_{x}+\mu\left(u_{x x}+u_{y y}-\frac{u_{y}}{R-y}\right)-\rho g, \\
\rho\left(u v_{x}+v v_{y}\right) & =-p_{y}+\mu\left[v_{x x}+v_{y y}-\frac{v_{y}}{R-y}-\frac{v}{(R-y)^{2}}\right] .
\end{aligned}
$$

At the wall boundary,

$$
u=-U_{b} \quad \text { and } \quad v=0, \quad \text { at } \quad y=0 \text {, }
$$

while at the gas-liquid interface

$$
\begin{aligned}
& u_{y}+v_{x}+\frac{2 h_{x}}{1-h_{x}^{2}}\left(v_{y}-u_{x}\right)=0, \quad \text { at } y=h(x), \\
& \text { and } p+\sigma \kappa+\mu h_{x}\left(u_{y}+v_{x}\right)-2 \mu v_{y}=0, \quad \text { at } y=h(x) .
\end{aligned}
$$

The unit normal vector at the interface has components

$$
\boldsymbol{n}=\frac{1}{\left(1+h_{x}^{2}\right)^{1 / 2}}\left(-h_{x}, 1\right)
$$

and the interface curvature is

$$
\kappa=\kappa_{1}+\kappa_{2}=\frac{h_{x x}}{\left(1+h_{x}^{2}\right)^{3 / 2}}+\frac{1}{(R-h)\left(1+h_{x}^{2}\right)^{1 / 2}} .
$$




\section{Appendix B: Liquid mass conservation}

In a stationary reference frame, the liquid swept out by the rising bubble must equal the sum of liquid flow rate in the tube and liquid flow rate within the film; in dimensional units:

$$
U_{b} \pi\left(R-h_{0}\right)^{2}=U_{l} \pi R^{2}-U_{f} \pi\left[R^{2}-\left(R-h_{0}\right)^{2}\right]
$$

where $U_{f}$ is the average velocity of the liquid in the uniform film region, which can be obtained by integrating the liquid velocity profile $u(y)$ in the uniform film region $C D$ :

$$
U_{f}=\frac{2}{R^{2}-\left(R-h_{0}\right)^{2}} \int_{0}^{h_{0}} u(y)(R-y) d y \text {. }
$$

In the uniform film region $\left(h_{x}=h_{x x}=0\right), u_{x}=u_{x x}=0, v=0$ and $p_{x}=0$ because the curvature of the interface is constant. Therefore, the $x$-momentum Eq. (A1b) simplifies as

$$
\mu\left(u_{y y}-\frac{u_{y}}{R-y}\right)=\rho g .
$$

This equation is integrated twice along $y$ with the boundary conditions

$$
\begin{aligned}
& u=0, \quad \text { at } y=0, \\
& \text { and } u_{y}=0, \quad \text { at } y=h_{0},
\end{aligned}
$$

thus leading to the following expression for the velocity profile in $C D$ :

$$
u(y)=\frac{\rho g R^{2}}{\mu}\left[\frac{y^{2}-2 R y}{4 R^{2}}+\frac{(1-H)^{2}}{2} \log \left(\frac{R}{R-y}\right)\right] .
$$

Substituting Eq. (B6) in Eq. (B2) and integrating gives:

$$
U_{f}=\frac{\rho g R^{2}}{\mu} \frac{1}{H(2-H)}\left[\frac{(1-H)^{4} \log (1-H)}{2}+\frac{(1-H)^{2}}{2}-\frac{1}{8}-\frac{3(1-H)^{4}}{8}\right],
$$

so that Eq. (B1) can be finally rearranged to express the bubble velocity as a function of the average liquid velocity in the tube and the dimensionless uniform film thickness:

$$
U_{b}=\frac{U_{l}}{(1-H)^{2}}+\frac{\rho g R^{2}}{\mu}\left[-\frac{(1-H)^{2} \log (1-H)}{2}-\frac{1}{2}+\frac{1}{8(1-H)^{2}}+\frac{3(1-H)^{2}}{8}\right] .
$$

The first term on the right-hand side of Eq. (B8) represents the contribution to the bubble motion given by the liquid flow rate within the tube, while the second term brings in the contribution of buoyancy. Since the term between square brackets is always positive, if 
buoyancy and liquid flow have the same direction, $g>0$ with the present notation, the bubble moves faster compared to the $g=0$ case, while for $g<0$ the bubble moves slower. A dimensionless form of Eq. (B8) can be derived by multiplying both the left- and right-hand side terms by $\mu / \sigma$, thus leading to Eq. (21) in the manuscript.

[1] M. Magnini, B. Pulvirenti, and J. R. Thome, "Numerical investigation of hydrodynamics and heat transfer of elongated bubbles during flow boiling in a microchannel," Int. J. Heat Mass Tran. 59, 451-471 (2013).

[2] Q. Zhang, S. M. Hassanizadeh, B. Liu, J. F. Schijven, and N. K. Karadimitriou, "Effect of hydrophobicity on colloid transport during two-phase flow in a micromodel," Water Resour. Res. 50, 7677-7691 (2014).

[3] S. Khodaparast, M. K. Kim, J. Silpe, and H. A. Stone, "Bubble-driven detachment of bacteria from confined micro-geometries," Environ. Sci. Technol. 51, 1340-1347 (2017).

[4] Y. Hu, S. Bian, J. Grotberg, M. Filoche, J. White, S. Takayama, and J. B. Grotberg, "A microfluidic model to study fluid dynamics of mucus plug rupture in small lung airways," Biomicrofluidics 9, 044119 (2015).

[5] S. M. H. Hashemi, M. A. Modestino, and D. Psaltis, "A membraneless electrolyzer for hydrogen production across the pH scale," Energ. Environ. Sci. 8, 2003-2009 (2015).

[6] J. R. Thome, V. Dupont, and A. M. Jabobi, "Heat transfer model for evaporation in microchannels. Part I: presentation of the model," Int. J. Heat Mass Tran. 47, 3375-3385 (2004).

[7] T. C. Thulasidas, M. A. Abraham, and R. L. Cerro, "Bubble-train flow in capillaries of circular and square cross section," Chem. Eng. Sci. 50, 183-199 (1995).

[8] M. T. Kreutzer, F. Kapteijn, J. A. Moulijn, C. R. Kleijn, and J. J. Heiszwolf, "Inertial and interfacial effects on pressure drop of Taylor flow in capillaries," AIChE J. 51, 2428-2440 (2005).

[9] M. Magnini, A. Beisel, A. Ferrari, and J. R. Thome, "Pore-scale analysis of the minimum liquid film thickness around elongated bubbles in confined gas-liquid flows," Adv. Water Resour. 109, 84-93 (2017).

[10] A. M. Bilek, K. C. Dee, and D. P. Gaver, "Mechanisms of surface-tension-induced epithelial cell damage in a model of pulmunary airway reopening," J. Appl. Physiol. 94, 770-783 (2003). 
[11] D. J. Nicklin, J. O. Wilkes, and J. F. Davidson, "Two-phase flow in vertical tubes," Trans. Inst. Chem. Eng. 40, 61-68 (1962).

[12] F. P. Bretherton, "The motion of long bubbles in tubes," J. Fluid Mech. 10, 166-188 (1961).

[13] G. I. Taylor, "Deposition of a viscous fluid on the wall of a tube," J. Fluid Mech. 10, 161-165 (1961).

[14] P. Aussillous and D. Quéré, "Quick deposition of a fluid on the wall of a tube," Phys. Fluid 12, 2367-2371 (2000).

[15] A. de Ryck, "The effect of weak inertia on the emptying of a tube," Phys. Fluid 14, 2102-2108 (2002).

[16] Y. Han and N. Shikazono, "Measurement of the liquid film thickness in microtube slug flow," Int. J. Heat Fluid Fl. 30, 842-853 (2009).

[17] M. Magnini, A. Ferrari, J. R. Thome, and H. A. Stone, "Undulations on the surface of elongated bubbles in confined gas-liquid flows," Phys. Rev. Fluid 2, 084001 (2017).

[18] R. Collins, F. F. de Moraes, J. F. Davidson, and D. Harrison, "The motion of a large bubble rising through liquid flowing in a tube," J. Fluid Mech. 89, 497-514 (1978).

[19] K. H. Bendiksen, "On the motion of long bubbles in vertical tubes," Int. J. Multiphas. Flow 11, 797-812 (1985).

[20] F. Fairbrother and A. E. Stubbs, "The bubble-tube method of measurement," J. Chem. Soc. 1, 527-529 (1935).

[21] D. T. Dumitrescu, "Strömung an einer luftblase im senkrechten rohr," Z. Angew. Math. Mech. 23, 139-149 (1943).

[22] R. M. Davies and G. I. Taylor, "The mechanics of large bubbles rising through extended liquids and through liquids in tubes," P. R. Soc. A 200, 375-390 (1950).

[23] A. O. Morgado, J. M. Miranda, J. D. P. Araujo, and J. B. L. M. Campos, "Review on vertical gas-liquid slug flow," Int. J. Multiphas. Flow 85, 348-368 (2016).

[24] A. M. F. R. Pinto, M. N. Coelho Pinheiro, S. Nogueira, V. D. Ferreira, and J. B. L. M. Campos, "Experimental study on the transition in the velocity of individual Taylor bubbles in vertical upward co-current liquid flow," Chem. Eng. Res. Des. 89, 1103-1110 (2005).

[25] S. Polonsky, L. Shemer, and D. Barnea, "The relation between the Taylor bubble motion and the velocity field ahead of it," Int. J. Multiphas. Flow 25, 957-975 (1999). 
[26] A. L. Hazel and M. Heil, "The steady propagation of a semi-infinite bubble into a tube of elliptical or rectangular cross-section," J. Fluid Mech. 470, 91-114 (2002).

[27] E. T. White and R. H. Beardmore, "The velocity of rise of single cylindrical air bubbles through liquids contained in vertical tubes," Chem. Eng. Sci. 17, 351-361 (1962).

[28] H. L. Goldsmith and S. G. Mason, "The movement of single large bubbles in closed vertical tubes," J. Fluid Mech. 14, 42-58 (1962).

[29] C. Lamstaes and J. Eggers, "Arrested bubble rise in a narrow tube," J. Stat. Phys. 167, 656-682 (2017).

[30] P. A. Kew and K. Cornwell, "Correlations for prediction of flow boiling heat transfer in smalldiameters channels," Appl. Therm. Eng. 17, 705-715 (1997).

[31] C. W. Hirt and B. D. Nichols, "Volume of fluid (VOF) method for the dynamics of free boundaries," J. Comput. Phys. 39, 201-225 (1981).

[32] K. Ruschak, "Flow of a falling film into a pool," AIChE J. 24, 705-709 (1978).

[33] J. Eggers, "Nonlinear dynamics and break up of free-surface flows," Rev. Mod. Phys. 69, 865-929 (1994).

[34] S. Khodaparast, N. Borhani, and J. R. Thome, "Application of micro particle shadows velocimetry ( $\mu \mathrm{PSV})$ to two-phase flows in microchannels," Int. J. Multiphas. Flow 62, 123-133 (2014).

[35] V. Ya. Shkadov, "Wave flow regimes of a thin layer of viscous fluid subject to gravity," Fluid Dyn. 2, 29-34 (1967).

[36] J. U. Brackbill, D. B. Kothe, and C. Zemach, "A continuum method for modeling surface tension," J. Comput. Phys. 100, 335-354 (1992).

[37] A. Ferrari, M. Magnini, and J. R. Thome, "A Flexible Coupled Level Set and Volume of Fluid (flexCLV) method to simulate microscale two-phase flow in non-uniform and unstructured meshes," Int. J. Multiphas. Flow 91, 276-295 (2017).

[38] S. V. Patankar, Numerical Heat Transfer and Fluid Flow (Hemisphere Publishing, New York, 1980).

[39] C.-W. Kang, S. Quan, and J. Lou, "Numerical study of a Taylor bubble rising in stagnant liquids," Phys. Rev. E 81, 066308 (2010).

[40] M. Heil, "Finite Reynolds number effects in the Bretherton problem," Phys. Fluid 13, 2517$2521(2001)$. 